\title{
Auferstehung und Epiphanie. Jenseits- und Körperkonzepte im Zweiten Makkabäerbuch
}

\author{
BARBARA SCHMITZ
}

Mit dem Zweiten Makkabäerbuch verbindet man in erster Linie die Vorstellung von der Auferstehung der Toten, wie sie im siebten Kapitel, der Erzählung von dem Martyrium der Mutter und ihrer sieben Söhne, aber auch an anderen Stellen zu finden ist. Neben der Auferstehung der Toten ist die Erzählung aber auch durchzogen von einem weiteren Vorstellungskomplex, den Epiphanien, von denen sich sechs Epiphanieschilderungen in der Erzählung finden. Beides sind Vorstellungskomplexe, die aus der diesseitigen erzählten Textwelt in ganz unterschiedlicher Form von einem Jenseits berichten. Beide Jenseitskonzepte operieren zudem, wenn auch je unterschiedlich, mit Körperkonzepten. Daher werden im Folgenden beide Denkfiguren im Einzelnen analysiert und in ihrer konzeptionellen Gestalt miteinander verglichen.

\section{1. „Auferstehung” im Zweiten Makkabäerbuch}

Das Zweite Makkabäerbuch gehört zu den frühen Zeugnissen vom Glauben an eine Auferstehung der Toten. ${ }^{1}$ Neben der Wiedererweckung der Totengebeine in Ez 37 und Erwartungen wie zum Beispiel auf ein Leben in Freude vor dem Angesicht Gottes (vgl. Ps 16,10-11) formulieren Dan 12,2 und Jes 26,192 den Gedanken an die Auferstehung der Toten. Von diesen Texten, die im Kontext von apokalyptischen Vorstellungen stehen, unterscheiden sich jene, die Auferstehung

1 Vgl. hierzu SCHUBERT, Entwicklung, 177-214; KELLERMANN, Überwindung, 259-282; LANG/MCDANNELl, Himmel; Bieberstein, Weg, 3-16; BEYERLE, Gottesvorstellung, 189-268; FISCHER, Tod; SETZER, Resurrection; NICKELSBURG, Resurrection.

2 Möglicherweise oder wahrscheinlich eine spätere Glosse in der sogenannten kleinen Jesajaapokalypse (Jes 24-27). Zur Diskussion vgl. WESTERMANN, Jesaja, 173-177; KELLERMANN, Gotteslob, 3-19. 
im Zweiten Makkabäerbuch ${ }^{3}$ thematisieren, nicht nur von ihrer literarischen Gattung, sondern auch von ihrer konzeptionellen Konstruktion: Während in ersteren Texten Auferstehung kollektiv als Auferstehung am Ende der Geschichte auf der Erde gedacht wird, ${ }^{4}$ geht es im Zweiten Makkabäerbuch um die Frage individueller Auferstehung und um himmlische Erhöhung besonderer Menschen nach ihrem Tod vor dem Ende der Geschichte. ${ }^{5}$

\section{1 „Auferstehung” in 2Makk 7}

Das siebte Kapitel des Zweiten Makkabäerbuchs schildert das Martyrium der Mutter und ihrer sieben Söhne, die von König Antiochus gezwungen werden, die väterlichen Gebote zu übertreten und Schweinefleisch zu essen (2Makk 7,1). Weil die Brüder und ihre Mutter aber eher bereit sind zu sterben, als die „väterlichen Gebote“ zu übertreten (2Makk 7,2), foltert und ermordet König Antiochus einen Bruder nach dem anderen. ${ }^{6}$ Vor ihrem Tod halten die Brüder und die Mutter Reden, in denen sie ihr bevorstehendes Martyrium theologisch deuten. Auferstehung wird dabei sowohl in der Rede des zweiten, dritten und vierten Sohnes (2Makk 7,9.11.14), als auch in den Reden der Mutter thematisiert (2Makk 7,23.29).

\subsection{1 „Auferstehung“ in der Rede des zweiten Sohnes (2Makk 7,9)}

In vier antithetischen Paaren erläutert der zweite Sohn in 2Makk 7,97 die Machtlosigkeit des als "Verbrecher" ( $\dot{\lambda} \alpha \dot{\sigma} \sigma \tau \omega \rho)$ bezeichneten syrischen Königs: Dabei stellt er dem König Antiochus Gott als den König der Welt gegenüber, das gegenwärtige Leben dem ewigen Leben, das

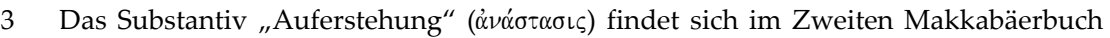
nur in 2Makk 7,14 und 12,43; das Verb „aufstehen“ ( $\alpha v^{\prime}(\sigma \tau \eta \mu \iota)$ in 2Makk 7,9.14; 12,44 (vgl. 14,33).

$4 \quad$ Vgl. hierzu auch Joh 6,30.40.44.54; 11,24 u.ö.

5 So z.B. in Bezug auf die Erzväter in TestBenj 10,6; vgl. Mk 12,26-27; in Bezug auf Ijob in Testljob 4,9; in Bezug auf die Märtyrer Offb 20,4-5. Neben diesen beiden Bedeutungen kann man noch eine weitere für „,aufrichten, aufwecken“ bzw. ,aufstehen“ finden: die Rückkehr eines Menschen in sein irdisches Leben (Jes 26,19; Mk 5,4; 9,27; Lk 16,31; Joh 11,23; Apg 9,49; Hebr 11,35). Vgl. hierzu auch KelLERMANN, Auferstanden, 64.

6 Vgl. hierzu auch TOMES, Heroism, 171-199.

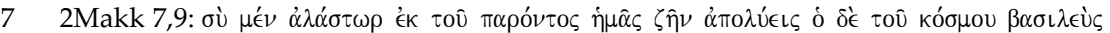

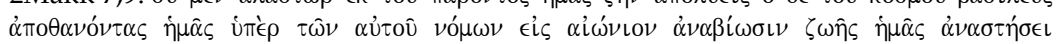
„Du Verbrecher nimmst uns zwar das gegenwärtige Leben. Der König der Welt aber wird uns, die wir für sein Gesetz sterben, zum Wiederaufleben in das ewige Leben erwecken." Die zitierten Passagen aus 2Makk sind in den meisten Fällen der Übersetzung von HABICHT entnommen, vgl. HABICHT, 2. Makkabäerbuch. 
Wiederaufleben dem Verlieren und das Auferwecktsein dem Sterben. Weil die Familie aber bereit sei, für Gottes Gebote zu sterben, werde Gott sie zu neuem Leben auferwecken. Gott ist das Subjekt der Auferweckung. Er wecke - so der zweite Sohn - die auf, die das Martyrium erleiden. Daher dürfen er, seine Brüder und seine Mutter dieses Wiederaufleben erwarten. Dies wird durch die dreimalige Wiederholung von "uns" ( $\dot{\eta} \mu \hat{\alpha} \varsigma$ ) betont. Das neue Leben nach dem Tod charakterisiert der zweite Sohn als ein Wiederaufleben $(\dot{\alpha} \nu \alpha \beta \dot{\omega} \omega \sigma \iota \varsigma)^{8}$ und als himmlisch-transzendentes Leben ${ }^{9}$ ohne zeitliche Befristung vor dem Ende der Geschichte. ${ }^{10}$

1.1.2 „Auferstehung“ in der Rede des dritten Sohnes (2Makk 7,11)

In der Rede des dritten Sohnes findet sich das Wortfeld "Auferstehung" nicht explizit. Am Beispiel seiner Hände (2Makk 7,11) ${ }^{11}$, die er dem König freiwillig neben seiner Zunge zum Martyrium ausstreckt, verdeutlicht er jedoch, dass er diese $(\tau \alpha \hat{v} \tau \alpha)$ gerne hinzuhalten bereit ist, weil er damit rechnet, sie von Gott wiederzuerlangen. Damit setzt der dritte Sohn voraus, dass das Leben nach dem Tod dem Leben vor dem Tod gleicht, und daher die Restitution einzelner Körperteile notwendig ist. Auf diese Weise wird eine über den Tod hinausgehende körperliche Identität bzw. Kontinuität postuliert. ${ }^{12}$ Der dritte Sohn stellt somit die Existenz nach dem Tod als eine körperliche dar.

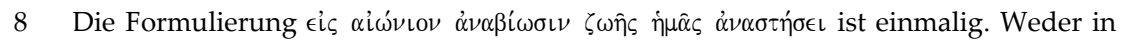
der LXX noch im NT findet sich der Ausdruck "Wiederaufleben“ ( $\alpha \nu \alpha \beta i \omega \sigma \iota \varsigma)$; zur Bedeutung und Verwendung im profangriechischen Kontext vgl. PAssOW, Handwörterbuch, 164. Auch Josephus spricht mit diesem seltenen Terminus vom Wiederaufleben der Seele nach dem Tod (vgl. Jos Ant XVIII 14). In Clem 19,4 wird mit diesem Terminus die himmlische Existenz nach dem Tod bezeichnet.

9 Zu dieser Formulierung siehe Mk 3,29; Hebr 9,12; vgl. HABICHT, 2. Makkabäerbuch, 234.

10 Vgl. GoldsteIn, II Maccabees, 305.

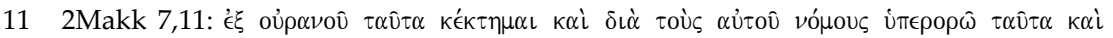

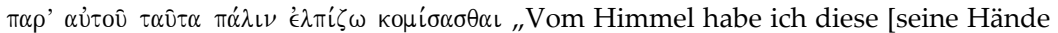
und Zunge, B.S.] erhalten, und um seines Gesetzes willen lasse ich diese fahren und hoffe diese von ihm wiederzuerlangen."

12 So auch ABEL, Les livres, 374. 
1.1.3 „Auferstehung“ in der Rede des vierten Sohnes (2Makk 7,14)

Die Rede des vierten Sohnes ist, wie die des zweiten, antithetisch ${ }^{13}$ aufgebaut $\left(2\right.$ Makk 7,14) ${ }^{14}$. Er betont, dass die Märtyrer auf eine Auferweckung durch Gott hoffen dürften, während für den König keine Hoffnung auf eine Auferstehung zum Leben bestehe. Interessant an dieser Rede ist, dass die "Auferstehung" nicht als sicheres Wissen präsentiert wird und dies auch nicht erwartet wird, sondern nur als Hoffnung geäußert werden kann.

\subsection{4 „Auferstehung“ in den Reden der Mutter (2Makk 7,22-23.27-29)}

Die erste Rede der Mutter ist an ihre ersten sechs Söhne gerichtet und wird in 2Makk 7,22-2315 erst im Nachhinein wiedergegeben. ${ }^{16}$ Dabei geht die Mutter von einer schöpfungstheologischen Reflexion aus, die in eine implizite Auferstehungstheologie mündet: Weil Gott als Schöpfer der Welt alles Leben ersonnen habe, werde er den Söhnen auch Le-

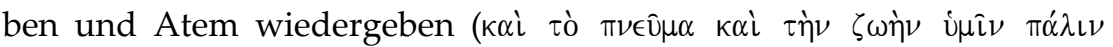

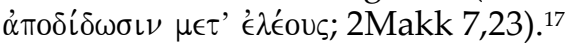

Die zweite Rede der Mutter (2Makk 7,27-29) ${ }^{18}$ ist an ihren siebten Sohn gerichtet. Diese weist eine ähnliche Logik wie die erste auf, indem

13 Mit dieser antithetischen Konstruktion markiert der vierte Sohn die Scharnierstelle zwischen den beiden Anliegen in den Reden der Brüder: Von der Reflexion über die „Auferstehung" leitet er zu den Aussagen der weiteren Brüder über, die den Tod von Antiochus thematisieren.

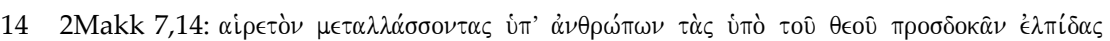

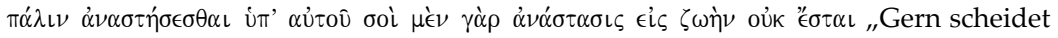
man aus dem Leben durch Menschenhand, wenn man dabei die Hoffnungen von Gott hegen darf, wieder von ihm aufgeweckt zu werden. Für dich aber gibt es keine Auferstehung zum Leben."

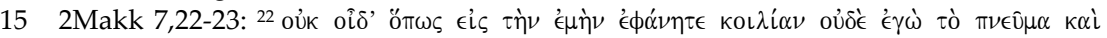

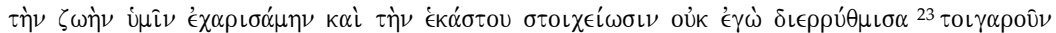

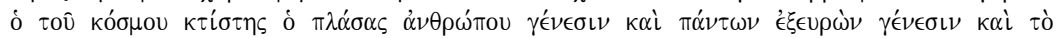

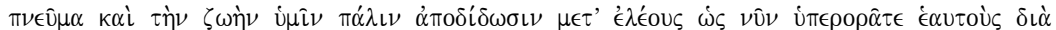

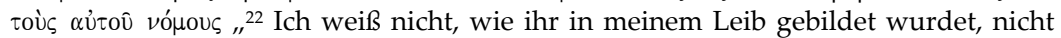
ich habe euch den Atem und das Leben geschenkt noch die Grundform eines jeden kunstvoll gebildet. ${ }^{23}$ Darum wird auch der Schöpfer der Welt, der das Menschengeschlecht bildet und das Werden aller Dinge ersinnt, Euch Atem und Leben in seinem Erbarmen wiedergeben, so wie Ihr jetzt Euer nicht achtet um seiner Gesetze willen."

16 So auch WACKER, Theologie, 261 sowie SCHMITZ, Geschaffen aus dem Nichts?.

17 Vgl. hierzu auch GoLDSTEIN, II Maccabees, 314.

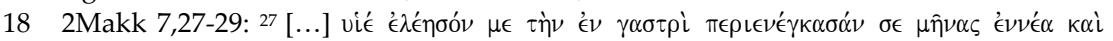

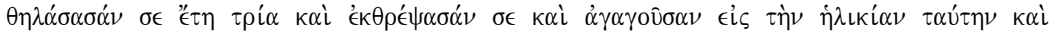

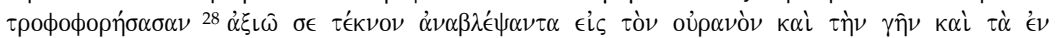

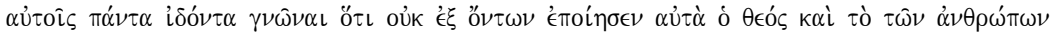

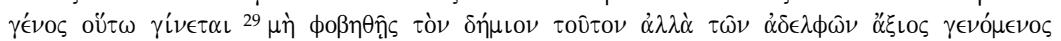


wieder Schöpfungstheologie den Ausgangspunkt der Argumentation bildet. So lenkt die Mutter den Blick von der Entstehung des individuellen Lebens und des Großwerdens ihrer Kinder auf den Anfang der ganzen Schöpfung, die allein auf Gott zurückgeht. Von diesen Anfängen schließt die Mutter in einem Analogieschluss auf das Leben nach dem Tod: Weil die Schaffung von etwas, das vorher noch nicht war, ${ }^{19}$ unfassbar und doch wirklich sei, sei das Leben nach dem Tod eine Neuschöpfung wie zu Beginn des Lebens. Aufgrund dieser Analogie kann die Mutter ihren jüngsten und letzten Sohn auffordern, dem Beispiel seiner Brüder zu folgen. Sie stellt ihm in Aussicht, dass sie ihn in der Zeit des Erbarmens mit seinen Brüdern wiederempfangen werde

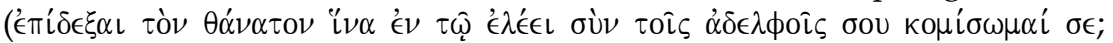
2Makk 7,29). Damit setzt sie voraus, dass sie und ihre Söhne aufgrund ihres Martyriums (vgl. 2Makk 7,41) auferstehen und sich wiedersehen werden.

Als Zeitpunkt für die Wiedererlangung des Lebens nennt die Mutter in beiden Reden das Erbarmen Gottes (' $' \lambda \in \sigma \xi ;$ 2Makk 7,23.27.29). Damit weist sie auf die Rede des ersten Sohnes zurück (2 Makk 7,6), der an die Zusage Gottes, dass er sich seines Knechtes erbarme, erinnert hat, die Gott Mose in Dtn 32,36 gegeben hat (vgl. auch Ps 135,14).20

Von der Erzählabfolge her nimmt die zweite Rede der Mutter inhaltlich die Rede des dritten Sohnes auf, ${ }^{21}$ der auch vom Wiedererlangen sei-

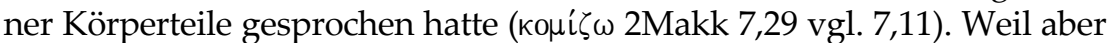
die Mutter ihre Rede vor allen ihren Söhnen gehalten hat, wird den Lesenden retrospektiv deutlich, dass die Argumentation des dritten Sohnes aus der Rede der Mutter inspiriert ist, die er zuvor gehört hat.

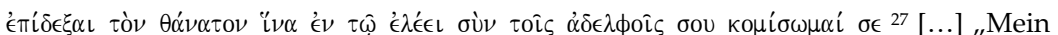
Sohn, hab Erbarmen mit mir, die ich dich neun Monate im Leib getragen und drei Jahre gestillt, bis zu diesem Alter für deine Ernährung und Erziehung gesorgt, mich um deine Pflege gekümmert habe. ${ }^{28}$ Ich bitte dich, Kind, blicke auf den Himmel und die Erde, sieh alles an, was in ihnen ist; so wirst du erkennen, dass Gott sie nicht aus Seienden gemacht hat und dass das Menschengeschlecht ebenso entsteht. ${ }^{29}$ Fürchte diesen Henker nicht, sondern nimm, deiner Brüder würdig, den Tod auf dich, damit ich dich beim Erbarmen mit deinen Brüdern wieder empfangen werde."

19 Vgl. hierzu ScHMITZ, Geschaffen aus dem Nichts?

20 Vgl. hierzu ARENHOEVEL, Theokratie, 160.

21 So auch BARTLETT, 2 Maccabees, 840. 
1.2 Weitere Auferstehungstexte im Zweiten Makkabäerbuch

Im Zweiten Makkabäerbuch wird Auferstehung an drei weiteren Stellen diskutiert: Im Fürbittgebet für getötete Soldaten in 2Makk 12,43-45, beim Selbstmord von Razi in 2Makk 14,46 sowie beim Traum von Judas Makkabäus in 2Makk 15,12-16.

\subsection{1 „Auferstehung“ in 2Makk 12,43-45: Die getöteten Soldaten}

Anlässlich eines Zwischenfalls im jüdischen Heer kommt die Rede noch einmal auf das Thema Auferstehung: Als bei Gefallenen heidnische Talismane gefunden wurden, sühnt man die Schuld dieser Kämpfer durch eine Kollekte und ein Opfer (2Makk 12,43-45).22 In der Erzählung wird nun auf der Metaebene explizit reflektiert, warum es sinnvoll sei, für Tote zu beten: Dies mache nur dann Sinn, wenn man tatsächlich mit der Auferstehung der Toten rechnet. Offenbar geht die Erzählung davon aus, dass nicht nur Märtyrer, sondern auch gefallene Soldaten, die mit Judas Makkabäus gekämpft haben, mit der Auferstehung belohnt werden. ${ }^{23}$

\subsection{2 „Auferstehung“ in 2Makk 14,46: Der toratreue Razi}

Ein weiteres Mal ist von Auferstehung in der Rede des toratreuen Razi die Rede, der, wie die Mutter und ihr dritter Sohn, von der Restitution der Körpers ausgeht. Vor seinem Suizid ruft Razi Gott, den Herrn über Leben und Tod, an, damit dieser ihm seine Körperteile ( $\tau \alpha \hat{v} \tau \alpha)$ wieder-

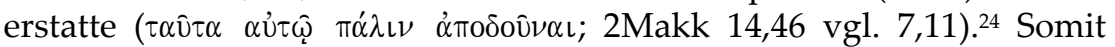
findet sich auch hier die Hoffnung auf himmlische Neuschöpfung, auch wenn das Wortfeld "Auferstehung" fehlt.

22 „Er tat gut und klug daran im Gedanken an die Auferstehung. Denn wenn er nicht erwartet hätte, dass die Gefallenen auferstehen würden, so wäre es überflüssig und töricht gewesen, für Tote zu beten. Auch zog er in Betracht, dass auf die herrlicher Lohn warte, die in Frömmigkeit sterben. Ein heiliger und frommer Gedanke! Daher verrichtete er für die Toten das Sühneopfer, damit sie von der Sünde erlöst würden“ (2Makk 14,44-45).

23 Vgl. hierzu auch GOLDSTEIN, II Maccabees, 450; zu den sprachlichen Schwierigkeiten in dieser Passage vgl. 445-447 sowie 447-448 zur weiteren Traditionsgeschichte des Fürbittgebets für Tote.

24 Ähnliches erzählt Josephus von essenischen Zeloten: „Freudig gaben sie ihr Leben in der Zuversicht, es wiederzuerlangen“ (Jos Bell 2,153). Vgl. zu diesem Abschnitt GOLDSTEIN, II Maccabees, 492-495. 
1.2.3 „Auferstehung“ in 2Makk 15,12-16: Die Fürbitte des toten Onias

Ein drittes Mal kommt das Thema "Auferstehung" implizit zur Sprache, als Judas von einem Traum berichtet, in dem ihm der Prophet Jeremia und der verstorbene Hohepriester Onias erschienen seien. ${ }^{25} \mathrm{In}$ ihm hält Onias, der als Märtyrer gilt (vgl. Dan 9,26; 2Makk 3-4; 1 Hen 90,8), für die Juden ein Fürbittgebet. Dieser Traumbericht zeigt, dass Onias nicht nur als Auferstandener geglaubt wird, sondern auch, dass er im weitesten Sinne körperlich identisch sein muss, denn sonst hätte Judas ihn nicht als Onias erkennen können.

\subsection{Fazit zu „Auferstehung“ im Zweiten Makkabäerbuch}

Im Zweiten Makkabäerbuch kristallisieren sich zum Thema "Auferstehung" zwei Felder heraus, die jeweils eigene Akzente setzen. Dies ist erstens die Vorstellung einer postmortalen himmlischen Erhöhung der Toten vor dem Ende der Geschichte. Diese Vorstellung findet sich in den beiden antithetisch strukturierten Reden des zweiten und vierten Sohnes (2Makk 7,9.14) sowie beim Fürbittgebet für die Toten (2Makk 12,43-45). Die zweite Vorstellung zum Thema Auferstehung ist die der Neuschöpfung und der Restitution des Körpers nach dem Tod. Diese postmortale Leiblichkeit wird in den beiden Reden der Mutter (2Makk 7,22-23.27-29), in der des dritten Sohnes (2Makk 7,11) sowie von Razi (2Makk 14,46) geäußert. Beide Vorstellungen finden sich im letzten Kapitel vereinigt: Beim Traum des Judas erscheinen Onias und Jeremia sowohl als post mortem Erhöhte, als auch als körperlich Restituierte (2Makk 15,12-16).

Das Thema "Auferstehung der Toten“ durchzieht somit das Zweite Makkabäerbuch als eine feine, unaufdringlich gezeichnete Linie. Dieser zurückhaltenden Thematisierung von Auferstehungsvorstellungen in der Erzählung korrespondiert die inhaltliche Aussage: Auferstehungsvorstellungen werden nicht in bunten, dick aufgetragenen Farben gemalt. Im Gegenteil: Man findet kaum detaillierte Beschreibungen von dem, was man sich unter "Auferstehung“ vorstellt. Lediglich im Modus der Hoffnung wird von einer neuen Qualität vom Leben nach dem Tod gesprochen, das man sich als leibliche Existenz und als Wiederaufleben vorstellt.

Die Leistungsfähigkeit der Vorstellung von der "Auferstehung", die theologisch durchaus diskutiert und umstritten war (vgl. Koh 3,1920 oder Jes Sir 41,3-4), zeigt sich im narrativen Setting der gesamten Er-

25 Jeremia starb nach Vita Prophetarum 14 auch als Märtyrer in Ägypten. 
zählung: Angesichts des gewalttätigen Auftretens einer fremden staatlichen Macht, die als radikale Bedrohung der eigenen theologischen, politischen und persönlichen Identität erfahren wird, ist die Denkfigur "Auferstehung" eine Möglichkeit, die als übermächtig empfundene staatliche Gewalt, der man ohnmächtig gegenüber steht, zu durchbrechen. Weil die Macht staatlicher Organe an der Grenze des Todes endet, eröffnet die Vorstellung von einem Leben nach dem Tod eine Freiheit, die zu neuen Handlungsspielräumen im Diesseits führt. Die Hoffnung auf eine Auferstehung der Toten ist daher die Möglichkeit, die Grenze zwischen Leben und Tod aus der Perspektive der in dieser Welt lebenden Menschen zu durchbrechen und damit der Tod bringenden, bedrohlichen Staatsmacht eine andere Macht, die Macht Gottes, entgegenzusetzen (vgl. die antithetische Gedankenführung in der Rede des zweiten und vierten Sohnes). Von Gott her, dem Schöpfer von allem Leben, entsteht eine verheißungsvolle Gegenwelt (vgl. die Reden der Mutter), die der Macht des Königs Antiochus entzogen ist, und gegenüber der sich der machtvoll auftretende, irdische König, als ohnmächtig erweist. Dabei sind die Aussagen über ein Leben nach dem Tod vorsichtig. Das, was nach dem Tod kommen mag, kann nur erhofft, niemals gewusst werden. Daher bleiben die Aussagen über das Leben nach dem Tod offen und vage.

\section{Epiphanieschilderungen im Zweiten Makkabäerbuch}

Die Grenze des Todes wird im Zweiten Makkabäerbuch aber nicht nur durch den Gedanken der Auferstehung der Toten aus der Perspektive der Menschen durchbrochen, vielmehr gibt es ein zweites Motiv, das von dem Durchbrechen dieser Grenze erzählt: Dies sind die Epiphanieschilderungen, von denen sechs sehr unterschiedliche $\mathrm{zu}$ finden sind

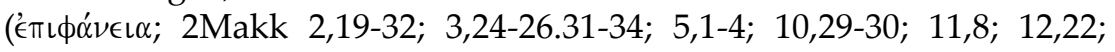
15,11-16.25-36). ${ }^{26}$ Ihre Bedeutung für die Erzählung zeigt sich darin, dass sie bereits im Vorwort explizit angekündigt werden (2Makk 2,21). $\epsilon \pi \iota \phi \alpha ́ \nu \in\left\llcorner\alpha\right.$ darf daher als ein „Schlüsselwort ${ }^{\star 27}$ der Erzählung gelten. ${ }^{28}$

26 "A 'manifest intervention' (epiphaneia) was a tangible event in which a supernatural being or force was perceived to act", GOLDSTEIN, II Maccabees, 192.

27 So HABICHT, 2. Makkabäerbuch, 187.

28 Im Zweiten Makkabäerbuch finden sich nicht sechs Epiphanieerzählungen, sondern die Erzählung ist auch von den mit diesem Wortfeld verbundenen Lexemen durch-

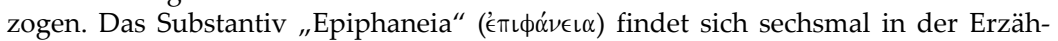

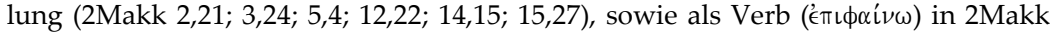

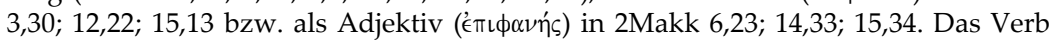

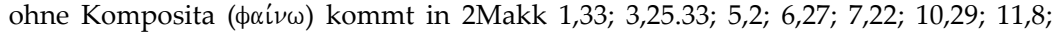

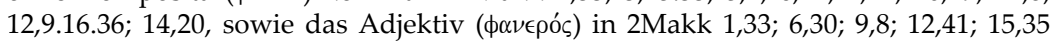


Exkurs: Epiphanien in der griechisch-hellenistischen Tradition

Epiphanien erzählen von Erscheinungen himmlischer Mächte in der diesseitigen Welt. Es ist immer wieder versucht worden, sie von den so genannten Theophanien ${ }^{29} \mathrm{zu}$ unterscheiden, von den Vorstellungen, die vom Kommen JHWHs mit gewaltigen Begleiterscheinungen und irdischem wie kosmischem Schrecken im Alten Testament handeln. Man kann zwischen beiden Termini differenzieren, auch wenn diese häufig synonym verwendet werden, ${ }^{30}$ um all jene Phänomene, die der Sache nach von der Erscheinung und dem Erfahrbarwerden einer Gottheit berichten, von jenen Darstellungen, in denen der Terminus „Epipha-

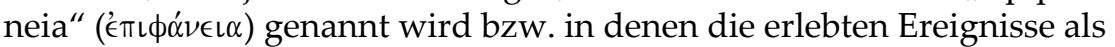
solche bezeichnet werden, ${ }^{31} \mathrm{zu}$ unterscheiden.

In der biblischen Literatur wird das Substantiv "Epiphanie"

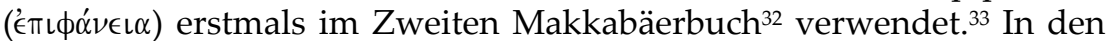
Schriften der Septuaginta, die hebräische Texte übersetzen, wird

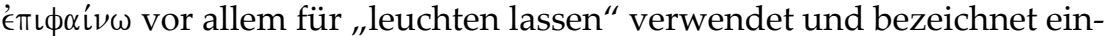
zelne Gottesvisionen und -auditionen (אור hif.; Num 6,25; Ps 30,17; 66,2; 79,4.8.20; 117,27; 118,135 [LXX]), wird aber auch als "sich zeigen“ (גלה; Gen 35,7; Ez 39,28), ,aufstrahlen“ (זרח; Dtn 33,2) sowie "sich finden lassen" (מצא nif; Jer 29,14) übersetzt. Interessanterweise gibt das Adjektiv

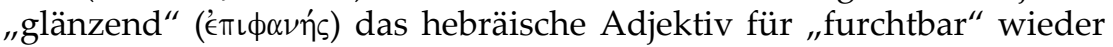
( נוֹר Ri 13,6; Jos 2,11; 3,4; Hab 1,7; Mal 1,14; 3,23), was dazu geführt hat, dass durch diese Übersetzung das Element des Furchtbaren in den Begriff der Epiphanie eingetragen worden ist. ${ }^{34}$

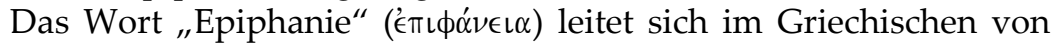
dem Verb "scheinen“ ( $\phi \alpha i \nu \omega)$ beziehungsweise von „,auf oder bei etwas

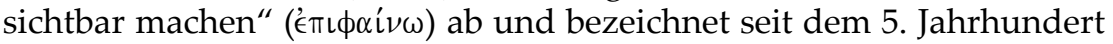
das äußerliche Erscheinen und die Erscheinungsweise. ${ }^{35}$ Dabei steht es

bzw. als Adverb ( $\phi \alpha \nu \in \rho \omega \hat{\omega})$ in 2 Makk 3,28 vor. Weitere Komposita aus dem Wortfeld

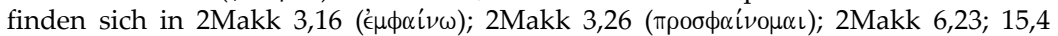

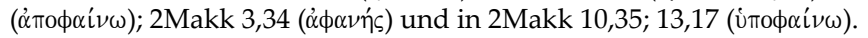

29 Vgl. hierzu JEREMIAS, Theophanie; SCRIBA, Geschichte; PfEIFFER, Jahwes Kommen; vgl. auch zu Theophanie in apokalyptischen Texten, BEYERLE, Gottesvorstellung, 51188.

30 WESTERMANN nimmt eine Unterscheidung beider Begriffe, allerdings nur aufgrund der unterschiedlichen Verwendung der Motive, vor, vgl. WESTERMANN, Lob, 69-75.

31 So auch OBERLINNER, Epiphanie, 552.

32 Zur Forschungslage vgl. WILLIAMS, Research, 69-83.

33 Einzige Ausnahme ist 2Sam 7,23 (LXX).

34 Zu Erscheinungserzählungen vgl. GUTTENBERGER, Gehalt, 40-63; 161-173.

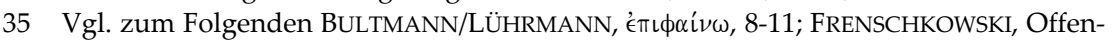

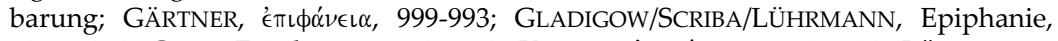

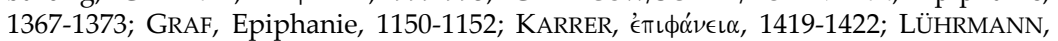


ursprünglich für ein plötzliches und unerwartetes Erscheinen. Es wird besonders in kriegerischen Kontexten verwendet, um das plötzliche Auftauchen eines Feindes zu beschreiben. ${ }^{36}$ Dieser ursprüngliche Gebrauch ist stets lebendig geblieben, sodass es neben der religiösen immer auch die profane Bedeutung des Wortes „Epiphanie“ gibt. Der religiöse Gebrauch ist erstmals auf einer Inschrift aus Kos belegt, in der die Niederlage der Gallier auf eine Epiphanie des Apollo zurückgeführt wird (278 v. Chr.; SIG 398). In dieser Verwendung ist noch der ursprüngliche militärische Kontext zu erkennen. Aber auch der erzählende Teil (Kolumne D) der Tempelchronik von Lindos (1. Jahrhundert v. Chr.) trägt den Titel émı $\alpha \nu \in \epsilon^{\prime} \alpha \iota$ (SIG 725). ${ }^{37}$ Epiphanien lösen bei den Menschen häufig Staunen, Verwunderung und Freude, aber auch Furcht aus. Berichte von Rettungen durch Epiphanien wurden häufig in Inschriften oder im Tempelarchiv festgehalten (so zum Beispiel in der Chronik von Lindos), die von der Macht des epiphanen Gottes ( $\theta \epsilon o ̀ \varsigma$

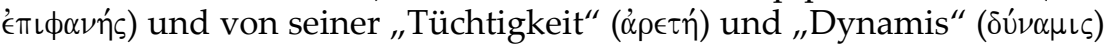
zeugen. ${ }^{38}$

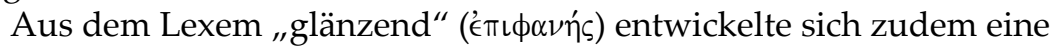
übertragene Bedeutung, um das "glänzende Aussehen" als Ansehen, Glanz und Würde einer Person zu bezeichnen. Daraus hat sich dann das sichtbare Erscheinen einer Person oder Gottheit entwickelt, das häufig mit der Vorstellung von Glanz und Leuchten verbunden bleibt. Dabei hat man sich das sichtbare Erscheinen nicht abstrakt vorgestellt, sondern immer konkret auf einen Gegenstand, ein Ereignis oder eine Person bezogen. Eine Epiphanie wird im persönlichen Erscheinen einer Gottheit erlebt, zeigt sich aber auch in außerordentlichen Begebenheiten. Epiphanie „bezeichnet also das Erscheinen der Gottheit weniger

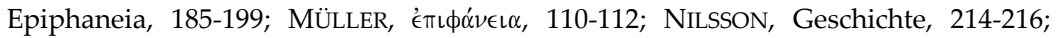
OBERLINNER, Epiphanie, 552-554; PAX, Epiphanie, 823-909; PAX, Epiphaneia; PFISTER, Epiphanie, 277-323; SÖDING, Epiphanie, 719-720; SPEYER, Hilfe, 55-77; VERSNEL, Ancient Man, 42-55; WACHSMUTH, Epiphanie, 1598-1601.

36 Zur militärischen Epiphanie vgl. PRITCHETT, Greek state, 11-47. In der antiken Mathematik bezeichnet Epiphanie auch eine zweidimensionale Fläche.

37 Vgl. die griechischen und hellenistischen Belege, aber auch die bei Josephus und

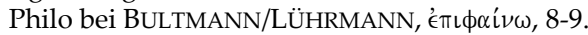

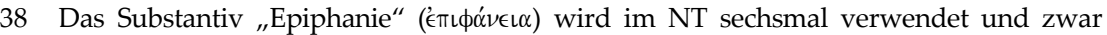
ausschließlich in der späteren Literatur (Tit 2,13; 1Tim 6,14; 2 Tim 1,10; 4,1.8; 2Thess

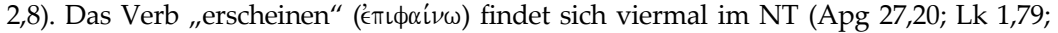

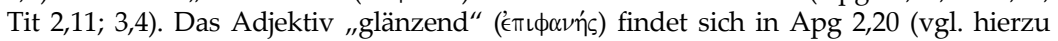

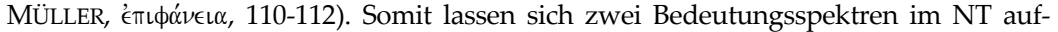
zeigen: Das Substantiv bezeichnet die erhoffte zweite Erscheinung des auferstandenen und erhöhten Christus am Ende der Zeiten (synonym zu „Parusie“), zum anderen wird mit dem Verb das Auftreten Jesu als „Epiphanie“ gedeutet. Vgl. hierzu DibeLIUS/ CONZELMANN, Pastoralbriefe; DONELSON, Pseudepigraphy; HASLER, Epiphanie, 193209; OBERLINNER, „Epiphaneia“, 192-312; ROLOFF, Brief, 352-355.358-365. 
im Mythos als in geschichtlichen Ereignissen“39. Auf diese Weise hat sich aus dem "Aufsehen erregenden Auftreten" einer Person im Hellenismus der Terminus Technicus des "Erscheinens" einer sonst verborgenen Gottheit entwickelt. Wie beim profanen Gebrauch meint Epiphanie auch hier die konkrete Erfahrung von Göttererscheinungen.

Die Hauptfunktion einer Epiphanie ist, dass die sichtbar erscheinende Gottheit zugunsten ihrer Verehrer rettend eingreift. ${ }^{40}$ In diesem religiösen Sinn hat die Epiphanie einer Gottheit soteriologische Funktion. Diese erweist die Gottheit in ihrem helfenden und rettenden Eingreifen im Kampf, indem sie aus einer Notsituation rettet oder zum Sieg verhilft. Daher gehören in diesen Vorstellungskomplex die Stich-

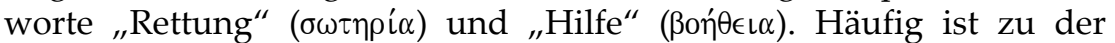
Gottheit um ihr rettendes Eingreifen gebetet worden. Epiphanien können aber auch Heilungen bewirken und mit Traumerzählungen oder Visionsschilderungen verbunden sein. ${ }^{41}$ Die unterschiedlichen Rettungen können dann zur Stiftung eines Kults führen und in einem Fest gefeiert werden.

Ende des Exkurses

\subsection{Die Ankündigungen von Epiphanien im Vorwort des Epitomators}

(2Makk 2,21-22)

Das Vorwort der Erzählstimme beginnt mit einem langen und syntaktisch raffiniert aufgebauten ersten Satz (2Makk 2,19-24), ${ }^{42}$ in dem alles für die Erzählung Wichtige zusammengefasst ist. Während das Subjekt „wir" ${ }^{\prime 3}$ und das Hauptsatzverb am Ende stehen (2Makk 2,24), beginnt

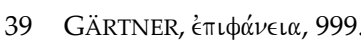

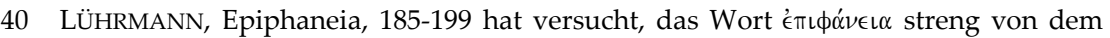
Begriff „Epiphanie“ zu unterscheiden, und will Ł̇ sondern als ",helfenden Eingreifen“ einer Gottheit verstanden wissen; dagegen positioniert sich VERSNEL, Ancient Man, 42-55.

41 Zu den Belegen vgl. PFISTER, Epiphanie, 292-297.

42 Es scheint eine Besonderheit der genuin auf Griechisch verfassten Septuagintaschriften zu sein, den jeweiligen Anfang mit einem langen und syntaktisch komplizierten Satz zu beginnen, vgl. Jdt 1,1; 8,1; Est 1,1 [LXX].

43 Interessant ist, dass das erste Subjekt, das in der Erzählung genannt wird, das „Wir" der Erzählstimme (2Makk 2,24) ist, das betont am Ende des ersten und langen Satzes der Erzählung steht. Das nächste Subjekt ist wieder das „Wir“ der Erzählstimme, das betont am Anfang des folgenden Satzes steht und damit diesen eröffnet. Nennung und Position der ersten Subjekte, die sich eben auf die Erzählstimme beziehen, lassen die Bedeutung der Erzählstimme eigens deutlich werden und sprechen für das "Selbstbewusstsein“ der Erzählstimme, die sich in ihrer Selbstpräsentation als „Epitomator" nur vermeintlich zurücknimmt, zumal er selbst sein eigenes Buch ge- 
der Satz mit den vier zentralen Themen beziehungsweise Figuren: Dies sind erstens Judas Makkabäus und seine Brüder, zweitens die Reinigung des Tempels und die Einweihung des Altars, drittens die Kriege gegen die Könige Antiochus Epiphanes und Eupator sowie viertens

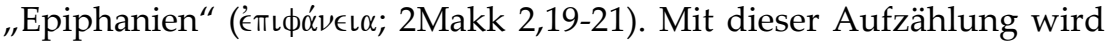
die folgende Handlung bereits von ihrem Ergebnis her resümierend vorweggenommen. Die „Epiphanien“ stehen in der Aufzählung her-

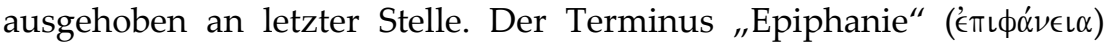
wird in 2Makk 2,21-22 vierfach expliziert. Erstens wird ihre Herkunft

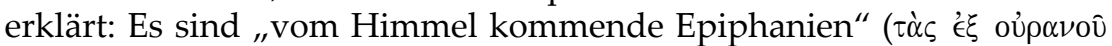

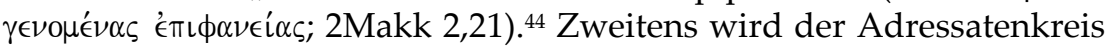
dieser Epiphanien genau festgelegt: Die Epiphanien werden „den

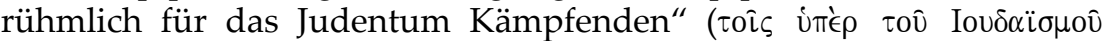
$\phi\llcorner\lambda o \tau i ́ \mu \omega \varsigma \dot{\alpha} \nu \delta \rho \alpha \gamma \alpha \theta \dot{j} \sigma \alpha \sigma \iota \nu ; 2$ Makk 2,21) zuteil.45 Damit wird bereits im Vorwort verdeutlicht, dass Epiphanien nicht von jedem wahrgenommen werden können, sondern an eine bestimmte Gruppe gebunden sind. Drittens wird das Ziel der Epiphanien thematisiert. Die, denen die Epiphanien erscheinen, sollen das ganze Land zurückerobern, die „Barbaren" verjagen, das Heiligtum wiedererlangen, die Stadt befreien und die Gesetze wieder aufrichten (2Makk 2,21-22). Mit dieser fünffach aufgefächerten Beschreibung werden die Erfolge, die aus den „Epiphanien" resultieren, ausführlich beschrieben. Viertens wird eine theologische Begründung dafür gegeben, warum sich diese Erfolge einstellen konnten: Der "Herr" (кúpıos) hat sich in seiner ganzen „Freundlichkeit" $\left(€ \pi L \in \epsilon^{\prime} \kappa \in L \alpha\right)^{46}$ „,erbarmend" 47 gezeigt. Damit wird der Bogen zur ersten Kategorie zurückgeschlagen: Die zu Beginn als „himmlisch“ eingeführ-

genüber dem schwer zu lesenden und unübersichtlichen Werk von Jason ausdrücklich lobt (2Makk 2,23-24).

44 2Makk 2,21-22: „sowie die Epiphanien, die den für die jüdische Sache voller Eifer und Tapferkeit Kämpfenden ( $\alpha \nu \delta \rho \alpha \gamma \alpha \theta \eta \dot{\sigma} \sigma \alpha \sigma \nu)$ zuteilgeworden sind, so dass sie trotz ihrer geringen Zahl das gesamten Land verheerten und die Massen der Barbaren verfolgten, ${ }^{22}$ dass sie weiter das in der ganzen besiedelten Welt berühmte Heiligtum zurückeroberten, die Stadt befreiten und den schon fast beseitigten Gesetzen wieder Geltung verschafften, da der Herr ihnen mit seiner ganzen Milde gnädig war."

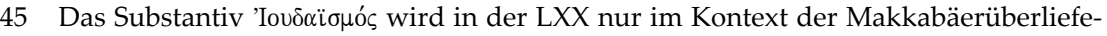
rung verwendet (2Makk 2,21; 8,1; 14,38 [zweimal]; 4Makk 4,26) und scheint eine

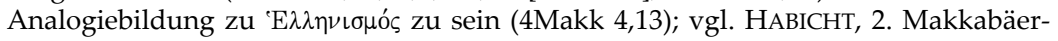
buch, 208.

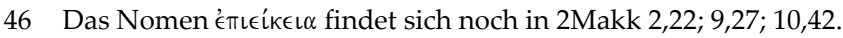

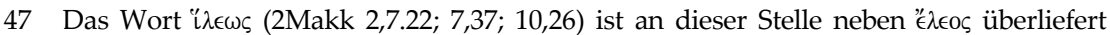
(vgl. HABICHT, 2. Makkabäerbuch, 206, mit Verweis auf die andere Lesart bei HANHART). In 2Makk findet sich das Wortfeld in 2Makk 2,18; 3,21; 7,27; 8,3; 9,13; 11,10 $\left(\epsilon^{\prime} \lambda \in \epsilon^{\prime} \epsilon\right)$ sowie in 2 Makk 4,37; 6,16; 7,23.29; 8,5.27 (' $\left.{ }^{\prime} \lambda \in O \varsigma\right)$. 
ten „Epiphanien“ zeugen von Gottes Freundlichkeit und seinem Erbarmen.

Im Vorwort werden zudem auch die Gegenspieler ${ }^{48}$ des „Herrn“ (кúpıos) eingeführt: König Antiochus (IV.) Epiphanes sowie sein Nachfolger, König Antiochus (V.) Eupator. Damit wird bereits der Kontrast zwischen dem König Antiochus, der im Vorwort explizit als „Antio-

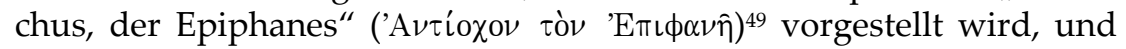
die Epiphanien eingeführt, die auf Gott zurückgehen.

Durch diese Einführung der Epiphanien im Vorwort wird ein programmatischer Rahmen für die folgenden Epiphanien etabliert. Daher sollen die folgenden sechs Epiphanieschilderungen unter den vier im Vorwort eingeführten Kategorien betrachtet werden: 1. Woher kommen die Epiphanien?, 2. Wer nimmt die Epiphanie jeweils wahr?, 3. Was wird gesehen? und 4. Was sind die Folgen des Gesehenen?

\subsection{Die erste Epiphanieschilderung: Die Heliodor-Episode}

(2Makk 3,24-26.33-34)

Die erste Epiphanieschilderung findet sich gleich zu Beginn der Erzählung (2Makk 3), ${ }^{50}$ in der aufgrund eines innerjüdischen Konflikts der königliche Gesandte Heliodor nach Jerusalem kommt und die Schatzkammer des Tempels betritt. Als er mit seinen Leibwächtern in die Schatzkammer des Tempels kommt, findet die erste Epiphanie statt: Zuerst erscheint ihnen ein Pferd in prächtigem Geschirr mit einem furchterregenden Reiter in einer Rüstung, die golden glänzt ( $\phi \alpha i \nu \omega)$. Das Pferd stürmt mit seinen Vorderhufen heftig auf Heliodor zu

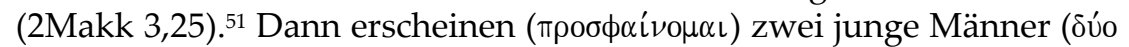
$\left.\nu \in \alpha v^{\prime}(\alpha L)\right)^{52}$, die von ausgezeichneter Körperkraft, herrlicher Erscheinung

48 So auch ENERMALM-OGAWA, langage, 130.

49 Dass diese Bezeichnung „Epiphanes“ ein Epitheton und nicht einfach nur Teil seines Namens ist, zeigen die weiteren Verwendungen in der Erzählung, an denen explizit

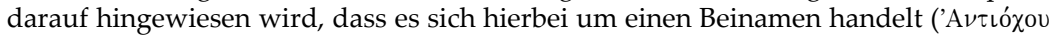

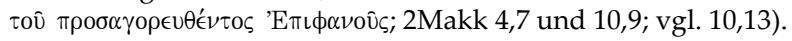

50 Die Erzählung um Heliodor gliedert sich in fünf Abschnitte (2Makk 3,1-3.4-7.8-23.2434.35-40).

51 2Makk 3,25:

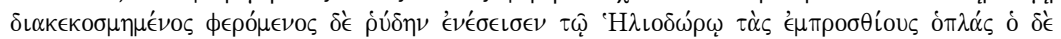

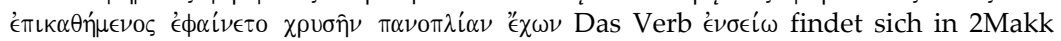
noch in 2Makk 12,15.37; 14,46. In der Schilderung werden zuerst die Erscheinenden, Pferd und Reiter, vorgestellt, anschließend deren Ausstattung mit prächtigem Geschirr und goldener Rüstung.

52 Momigliano sieht hier Bezüge zur Chronik von Lindos, vgl. Momigliano, Wisdom, 98-99. Überlicherweise wird auch die Verwandtschaft der beiden jungen Männer zu den Dioskuren, jenem unzertrennlichen Zwillingspaar betont, das in 
und kostbar gekleidet sind (2Makk 3,26). Diese jungen Männer schlagen Heliodor, der daraufhin zu Boden stürzt.

Als Urheber dieser Epiphanie wird explizit Gott genannt, der diese

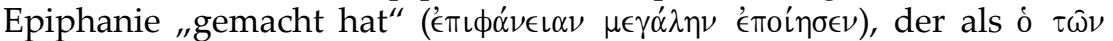

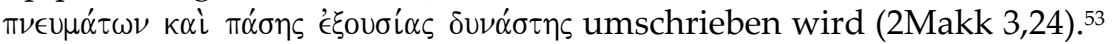
Die vorsichtigen Formulierungen in 2Makk 2,25.33 scheinen deutlich zu machen, dass die Epiphanie nur von Heliodor und seinen Männern gesehen wurde. ${ }^{54}$ Die Reaktion auf die Dynamis Gottes ist Erschrecken

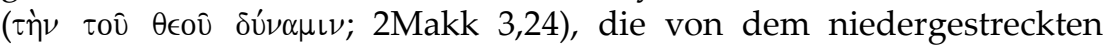
Heliodor erkannt wird, der hilflos auf einer Bahre aus der Schatzkam-

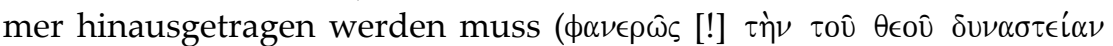
є̇ $\epsilon \nu \omega \kappa o ́ \tau \epsilon \varsigma ; 2 M a k k ~ 3,28)$. Dass der mächtige seleukidische Gesandte nun ohne Stimme, Hoffnung und Rettung ( $\sigma \omega \tau \eta \rho i \alpha)$ daniederliegt (2Makk 3,29), wird auf die göttliche Energeia ( rückgeführt. So endet die erste Epiphanieschilderung für die Feinde Gottes mit Furcht und Schrecken, für Israel aber mit Freude und Heiterkeit, weil der Kyrios Pantokrator in der Epiphanie erschienen sei (

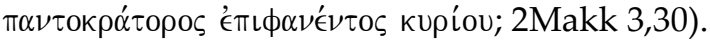

Nach dieser ersten Epiphanie folgt eine zweite, deren Empfänger wieder Heliodor ist: Auf Bitten der Vertrauten des Heliodor, Onias mö-

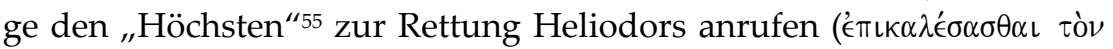

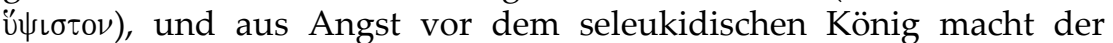
Hohepriester Onias ein Opfer zur Rettung ( $\sigma \omega \tau \eta \rho i ́ \alpha)$ des sterbenden

Notsituation rettend eingreift und als deren Namen Kastor und Pollux überliefert sind, vgl. FISCHER, Heliodor, 131 vgl. auch 126. Zu weiteren Bezügen zur paganen Literatur vgl. DORAN, Temple Propaganda, 48.

53 Beide Begriffe, $\pi \nu \in \hat{\nu} \mu \alpha$ und ' $\xi \xi o v \sigma i \alpha$, werden in der Erzählung nur an dieser Stelle auf Gott bezogen. Auch wenn es ähnliche biblische Formulierungen gibt (vgl. Num

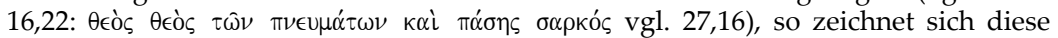
Formulierung gerade dadurch aus, dass der Begriff "Gott" nicht fällt; zur Formulierung vgl. auch GOLDSTEIN, II Maccabees, 212-213.

54 So auch SCHWARTZ, 2 Maccabees, 201: "Such care has two advantages: it allows the author, who might worry about rationalistic critics, to evade an unequivocal statement that a miracle occurred; and it allows for the continuation of the story, which depends upon Simon being able to claim that the attack was staged (4:1)".

55 Diese Bitte ist deswegen besonders interessant, weil die Bitte der seleukidischen Begleiter, den „Höchsten“ anzurufen, auf keinen Gott hin spezifiziert. Im Kontext der theologischen Tendenzen in seleukidischer Zeit werden die unterschiedlichen (polytheistischen) Systeme in gegenseitiger Äquivalenz verstanden, sodass aus der Perspektive der Begleiter von Heliodor der im Jerusalemer Tempel verehrte „Höchste“ äquivalent mit ihrem "Höchsten“, mit Zeus, ist, dessen Kult unter der Regentschaft von Antiochus IV. besondere Aufmerksamkeit von staatlicher Seite zukam. (vgl. 2Makk 6,2), vgl. hierzu FISCHER, Heliodor, 132. Zu dem Terminus vgl. auch ZIMMERMANN, Namen, 573-587. 
Heliodor (2Makk 3,31-32). ${ }^{56}$ Während des Opfers erscheinen Heliodor

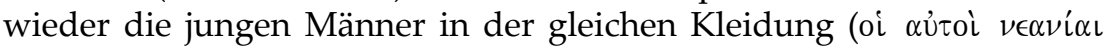
$\pi \alpha \dot{\lambda} \iota \nu \dot{\epsilon} \phi \dot{\alpha} \nu \eta \sigma \alpha \nu)$. Sie verpflichten Heliodor zu Dank gegenüber dem Hohepriester Onias und verlangen, dass Heliodor die große Macht

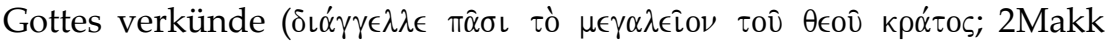
3,33-34). Auf diese Weise endet die erste, doppelt gestaltete Epiphanieschilderung mit der aus der Epiphanie stammenden Aufforderung zur Verkündigung der großen Macht des Herrn aus dem Mund eines Hei-

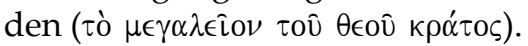

Die erste Epiphanieschilderung ist durch Signalworte geprägt: In 2Makk 3 findet sich die dichteste Verwendung des Wortfelds ",erscheinen" innerhalb der ganzen Erzählung. ${ }^{57}$ Zugleich finden sich in dieser ersten Epiphanieerzählung alle wesentlichen Motive, die in den folgenden wiederkehren werden, wie Pferde, Reiter, Waffen, Gold, das Motiv des Fürchtens usw. ${ }^{58}$ Auf diese Weise wird in der ersten Epiphanieschilderung ein Motivrepertoire etabliert, das in den weiteren variiert und modifiziert wird.

Zudem zeigt sich Gott bereits hier ${ }^{59}$ seinem Volk als der „epiphane“ Gott und Retter. Damit ist die Heliodor-Erzählung keine „(für den Fortgang der Ereignisse folgenlose) Vorgeschichte“"60, sondern bildet als

56 BICKERMANN identifiziert Version A (24.25.27.28.30) und Version B (26.29.31-36) der Epiphanieerzählung, vgl. BICKERMANN, Héliodor, 7-40; wieder abgedruckt in erweiterter Fassung in: BICKERMANN, Studies, 159-191. Dies übernimmt auch MARIANO, L'episodio, 421-456.

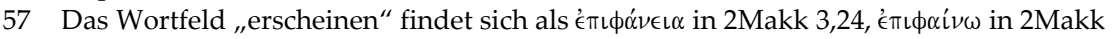

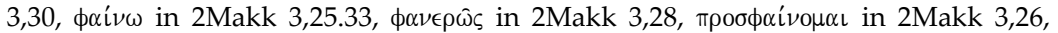

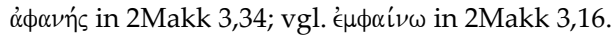

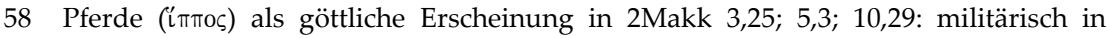
2Makk 5,2; 10,24; 11,2; 15,20; (' $\epsilon^{\prime} \phi$ เाто)): 11,8 (himmlisch); 12,35 (militärisch) sowie

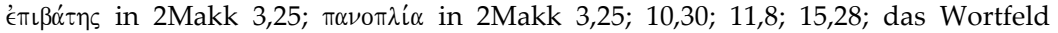
"golden" (Хрuбôิs) in 2Makk 3,25; 5,2.3; 10,29; 11,8; 15,15 (sonst in 2Makk 2,2; 3,11;

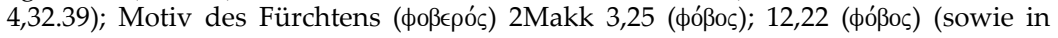

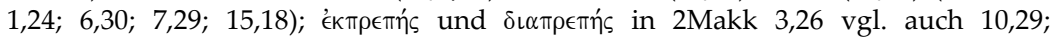
$\mu \in \gamma \alpha \lambda 0 \pi \rho \epsilon \pi \omega \varsigma$ in 15,13 sowie $\pi \rho \epsilon \pi o ́ v \tau \omega \varsigma$ in 15,12. Das Wortfeld findet sich im 2. Makkabäerbuch nur im Superlativ und rahmt die erste und die letzte Epiphanie $(\kappa \alpha ́ \lambda \lambda \iota \sigma \tau \alpha)$ 2Makk 3,1.25.26; 15,12.17.

59 Die Deutung der Ereignisse als Epiphanie Gottes, die auch Heliodor vor seinem König vertritt, werden von Simeon, der die Auseinandersetzung ausgelöst hat, bestritten: Simeon behauptet, dass Onias die "Epiphanie“ selbst inszeniert habe (2Makk 4,1). Seine Position wird von der Erzählstimme als "Verleumdung" (какодоү'є $\omega$ ) deutlich abgewertet, doch erweist sich diese Deutung Simeons als so wirkmächtig, dass sie den König erreicht und sich Onias deswegen zum König begibt (2Makk 4,5-6).

60 So ENGEL, Bücher, 322. 
Episode ${ }^{61}$ den Hintergrund in der noch als ideal und golden geschilderten Zeit (2Makk 3,1-3). Die unter dem neuen König Antiochus IV. folgenden Ereignisse stehen damit unter dem Eindruck der ersten Epiphanieschilderung, die von der Rettung des Heiligtums und der Ver-

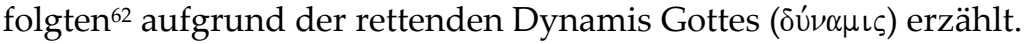

\subsection{Die zweite Epiphanieschilderung: Himmlische Phänomene in der Stadt (2Makk 5,2-4)}

Die zweite Epiphanieschilderung findet sich im Kontext des zweiten Ägyptenfeldzugs von Antiochus IV. im Jahre 168 v. Chr. (2Makk 5,1).

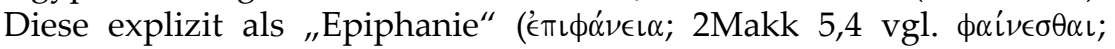
2Makk 5,2) bezeichnete Schilderung (2Makk 5,2-4) berichtet, dass man in der Stadt ungefähr vierzig Tage ${ }^{63}$ lang ein großes Heer von Pferden,

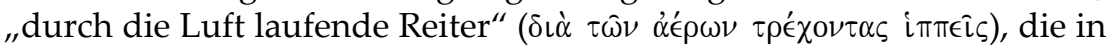
2Makk 2,21 explizit angekündigt werden, und Soldaten in golddurch-

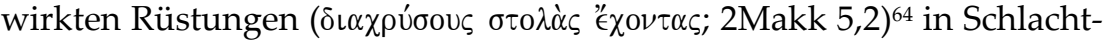
ordnungen habe sehen können. Diese seien mit vielen verschiedenen Waffen ausgestattet (2Makk 5,2-4) gewesen. ${ }^{65}$

61 So auch BARTLETT, 2 Maccabees, 834. Der literaturwissenschaftliche Terminus

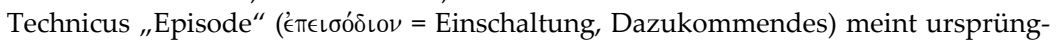
lich eine im antiken Drama zwischen die Chorgesänge eingeschobene Handlung und bezeichnet eine in eine Haupthandlung eingeschobene und mit ihr meist nur locker verknüpfte Nebenhandlung (subplot), deren Funktion es ist, die Haupthandlung hervorzuheben, zu ihrem Verständnis beizutragen oder die Handlung zu erweitern. Ihr Bezug zur Haupthandlung oder -figur kann parallel oder antithetisch strukturiert sein; vgl. ARNOLD/SINEMUS, Grundzüge, 467-468; WILPERT, Episode, 228; SCHWEIKLE, Episode, 131; MARTINEZ/SCHEFFEL, Einführung, 110-111; vgl. WERTHEIM, Fabel, 87-107, vgl. auch die Differenzierungen bei LÄMMERT, Bauformen, 260-261.

62 So auch FISCHER, Heliodor, 123.

63 Mit der Erwähnung der vierzig Tage wird eine biblisch vielfach besetzte Zahl genannt, die betont als Motiv verwendet wird, was die Formulierung „ungefähr" deutlich macht: Es handelt sich nicht um eine exakte Zahlenangabe, die die Dauer der Epiphanie angeben soll, sondern die Erzählung macht selbst auf ihre Intention, auf die Symbolik der Zahl Vierzig anzuspielen, aufmerksam und will damit ideale Vollständigkeit insinuieren.

64 Soldaten in goldener Rüstung finden sich im Zweiten Makkabäerbuch nur in Epiphanieschilderungen (2Makk 3,25; 5,2.3; 10,29; 11,8).

65 2Makk 5,2-4: , "2 Und es geschah, dass man in der ganzen Stadt fast vierzig Tage lang Reiter in golddurchwirkten Rüstungen durch die Luft sprengen sah, weiter Abteilungen von Lanzenträgern, die scharweise verschieden bewaffnet waren, ${ }^{3}$ sowie Scharen von Pferden in Schlachtordnungen, ferner Angriffe und Attacken der einen die der anderen, Bewegungen der Schilde, einen Wald von Speeren, gezückte Schwerter, geschleuderte Geschosse, das Funkeln des Zierrats und mannigfaltige Panzerungen. ${ }^{4}$ Daher beteten alle, die Epiphanien mögen von guter Vorbedeutung sein." 
Bei dieser Epiphanie bleibt offen, wer der Sender und wer der genaue Empfänger ${ }^{66}$ dieser Epiphanie ist. Auch die Stadt, in der die Epiphanie stattfindet, wird nicht explizit mit ihrem Namen benannt. ${ }^{67}$ Zudem bleibt bei dieser Epiphanie offen, ob es sich bei diesem „ambi-

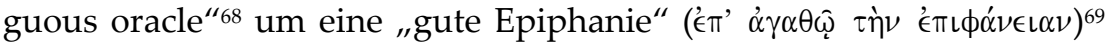
handelt, weil derartige Zeichen meist als Ankündigung von Unglück verstanden werden. ${ }^{70}$ Deswegen wird betont, dass die Empfänger nur

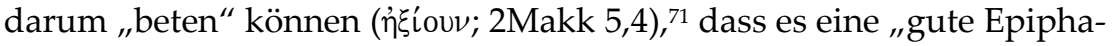
nie" sei. Damit unterscheidet sich diese Epiphanie grundlegend von der ersten.

Die Funktion dieser Epiphanie ist umstritten: Die Versuche, für die Epiphanie eine „natürliche“ Erklärung zu finden, gehen an der Erzählung vorbei. ${ }^{72}$,The meaning of this apparition was uncertain, apparently, but the author of 2 Maccabees is certainly indicating that this moment marked the arrival of the crisis for Jerusalem." 73

Nach dieser „eher ominösen "74 Epiphanie wird im weiteren Handlungsablauf erzählt, dass Antiochus die Stadt Jerusalem eingenommen hat und ein unglaubliches Blutbad und Gemetzel unter der Bevölkerung anrichtet. Als Höhepunkt seiner Greueltaten betritt er unter Mitwirkung des Hohenpriesters Menelaos den Tempel von Jerusalem und entweiht diesen (2Makk 5,11-16). Die Erzählung wird in 2Makk 5,17-20

66 Am Ende der Epiphanieschilderung werden die Bewohner der Stadt unter dem offenen Begriff „,alle“ subsumiert ( $\pi \dot{\nu} \nu \tau \epsilon \varsigma ; 2$ Makk 5,4).

67 Es dürfte sich dabei um Jerusalem handeln. Jerusalem wird im Zweiten Makkabäerbuch immer wieder ohne Eigenname nur als "die Stadt" ( $\dot{\eta}$ mó $\lambda \iota \varsigma$ ) bezeichnet (2Makk 2,$22 ; 3,1.4 ; 4,2$ u.ö.).

68 SCHWARTZ, 2 Maccabees, 253, vgl. 251-252.

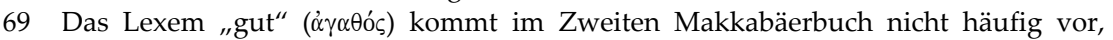
meist im Kontext von Epiphanien (2Makk 5,4; 15,11.23).

70 Vgl. TEDESCHE/ZEITLIN, Second Book of Maccabees, 144; DOMMERSHAUSEN, 1 Makkabäer, 125.

71 Dabei ist auch unklar, wen sie bitten, dass es eine gute Epiphanie sei. Das Verb „bitten" ( $\dot{\alpha} \xi$ เó $\omega)$ hat hier kein Objekt und wird im Zweiten Makkabäerbuch sowohl im profanen zwischenmenschlichen Kontext (vgl. 2Makk 3,31; 4,19; 7,28; 9,26; 11,17.24), als auch in Bezug auf Gott verwendet. Wenn aber Gott der Adressat der Bitte ist, dann wird er stets als Objekt explizit benannt (vgl. 2Makk 8,14.29; 10,4.16.26).

72 Vgl. z.B. GoldSTEIN, II Maccabees, 247: "The 'miraculous' apparitions are all explainable by mirages projected from Antiochus' army because of the presence of a strong atmospheric temperature inversion, in which cold denser air overlies warm lighter air. The phenomena called 'looming' or 'towering' would lead to images of the distant army being perceived as in the air and near, and the phenomenon of a 'double superior mirage', the lower image inverted, the upper one erect, would account for the charges and countercharges".

73 BARTLETT, Book, 257. "The author is suggesting that this was a time of crisis, in which divine intervention was the only hope", BARTLETT, 2 Maccabees, 837.

74 So FRENSCHKOWSKI, Offenbarung, 106. 
durch einen ausführlichen Kommentar unterbrochen, in dem reflektiert wird, warum Antiochus nicht, wie zuvor Heliodor, durch eine Epiphanie Gottes gestoppt und bestraft worden sei. Offensichtlich erscheint es als notwendig zu erklären, warum Gott, der hier im Kontrast

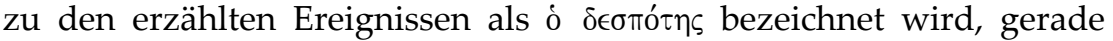
nicht eingreift und das Schlimmste verhindert. Die Erzählstimme führt Gottes Nicht-Eingreifen auf die „Verfehlung derer, die die Stadt be-

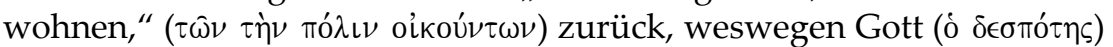
zürnt, wenn auch nur für kurze Zeit (2Makk 5,17). Hätten sich die

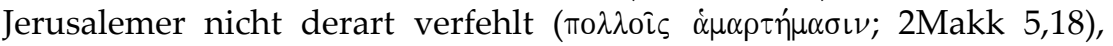
hätte Gott, wie bei Heliodor, sofort eingegriffen - so der Kommentar. Darauf folgt der - meines Erachtens - wichtigste und zentral(st)e Satz des Zweiten Makkabäerbuchs: „Aber der Herr hatte nicht wegen der Stätte das Volk, sondern um des Volkes Willen die Stätte auserwählt" (2Makk 5,19). ${ }^{75}$ Im Zweiten Makkabäerbuch geht es nicht, wie meist angenommen wird, um den Tempel, sondern um das Volk. ${ }^{76}$

\subsection{Die dritte Epiphanieschilderung: Himmlisches Eingreifen zugunsten von Judas (2Makk 10,29-30)}

Ein drittes Mal findet sich himmlisches Eingreifen ${ }^{77}$ im Kampf zwischen Judas Makkabäus und Timotheus (2Makk 10,24-38):78 Als bei Sonnenaufgang beide Truppen in der Nähe von Jerusalem aufeinander treffen und der Kampf bereits heftig entbrannt ist, erscheinen (' $\epsilon \phi \alpha ́ v \eta \sigma \alpha \nu$; 2Makk 10,29) vom Himmel fünf ${ }^{79}$ prächtig gekleidete Männer auf Pfer-

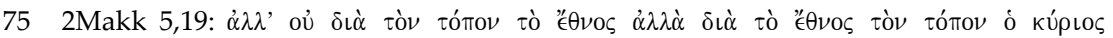
$\dot{\epsilon}^{\prime} \xi \in \lambda^{\prime} \dot{\epsilon}^{\prime} \xi \alpha \tau$.

76 So auch SCHWARTZ, 2 Maccabees, 46.

77 Auch wenn die epiphane Unterstützung von Judas nicht explizit als „Epiphanie“ bezeichnet wird, wird durch die verwendeten Motive, die Einführung der himm-

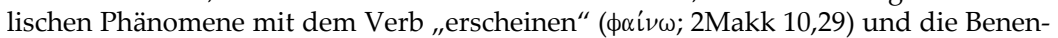
nung der Erscheinungen als „vom Himmel“ deutlich, dass diese von Gott stammen ( $\xi \xi$ oủp $\alpha \nu$ ovi; 2 Makk 10,29 vgl. die "himmlischen Epiphanien“ in 2Makk 2,21 sowie $3,34 ; 11,10 ; 15,34)$.

78 2Makk 10,29-30: "29 Und als der Kampf heftig tobte, zeigten sich den Feinden vom Himmel fünf prächtige Männer auf Pferden mit goldenen Zügeln und machten sich zu Anführern der Juden. ${ }^{30}$ Zwei von ihnen nahmen Judas in ihre Mitte, beschirmten ihn mit ihren Rüstungen und bewahrten ihn vor Verletzungen, gegen die Feinde aber schleuderten sie unentwegt Pfeile und Blitze. Deshalb stoben die Feinde, von Finsternis überschüttet und voll Verwirrung, auseinander."

79 ABEL mit Verweis auf GRIMM (Buch der Maccabäer) vermutet, dass die fünf Erscheinenden auf die fünf Brüder der Makkabäer oder mit Verweis auf GINZBERG (Legends, 241) auf Abraham, Isaak, Jakob, Mose und Aaron zurückzuführen seien (ABEL, Les livres, 415). 


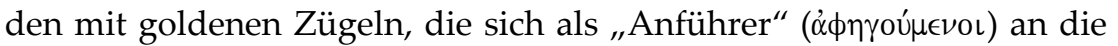
Spitze der Truppen stellen. Zwei ${ }^{80}$ von ihnen flankieren Judas und schleudern Pfeile und Blitze gegen die Feinde (2Makk 10,30; vgl. Ps 144,6). Als Adressat dieser Erscheinung werden ausdrücklich die Geg-

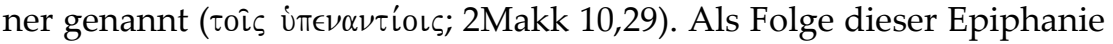
stieben die Feinde auseinander. Sie sind von Blindheit geschlagen und fliehen voller Verwirrung (2Makk 10,30). 20500 Mann und 600 Reiter werden erschlagen (2Makk 10,31).

\subsection{Der Rekurs auf die Geschichte Israels und die Sanherib-Erzählung}

(2Makk 8)

Gerade vor dem Hintergrund der dritten Epiphanie in 2Makk 10,29-30 sowie der expliziten Thematisierung der Epiphanielücke in 2Makk 5,17-20 stellt sich die Frage, ob nicht auch in der Schlacht mit Nikanor in 2 Makk $8^{81}$ eine Epiphanie „fehle“.

In der ersten großen militärischen Auseinandersetzung findet sich tatsächlich keine Epiphanie. ${ }^{82}$ Aber vor der Schlacht mit Nikanor erzählt Judas ausgewählten Kämpfern von Rettungen, die sie schon in der Geschichte von Gott erfahren haben, um ihnen die Hilfe Gottes deutlich zu machen, die ihnen bereits früher vom Himmel zuteil ge-

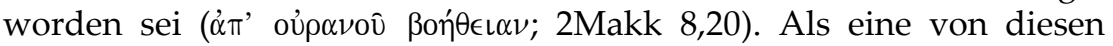
nennt Judas die wundersame Rettung Jerusalems zur Zeit Hiskijas während der Belagerung Jerusalems durch Sanherib $\left(2\right.$ Makk 8,19), ${ }^{83}$ die man in der theologischen Reflexion auf einen "Boten" oder "Engel" Gottes zurückgeführt hat (2Kön 19,35; Jes 37,36; vgl. 2Chr 32,21). Die Erinnerung an die Rettung vor den Truppen Sanheribs scheint sich deshalb besonders zu eignen, weil Judas mit ihr auf eine alte Erzählung zurückgreift, in der das Rettungshandeln Gottes auf nicht-irdische Gestalten zurückgeführt wird. ${ }^{84}$ Die Erinnerung an Sanherib ist aber deut-

80 Zur Lesart vgl. HABICHT, 2. Makkabäerbuch, 253 sowie HANHART, Text, 450; vgl. auch SCHWARTZ, 2 Maccabees, 388.

81 Zu 2Makk 8 vgl. auch NICKLAS, Geschichte, 25-41.

82 Diese Verbindung stärkt zudem die Vermutung, dass es sich bei beiden Nikanors um dieselbe Figur handeln dürfte, vgl. hierzu HABICHT, 2. Makkabäerbuch, 239.

83 2Makk 8,19: „Er zählte ihnen des Weiteren die Hilfeleistungen auf, die es zur Zeit der Vorfahren gegeben hatte, sowie die gegen Sanherib, als 185.000 Mann zugrunde gingen, ...".

84 Zudem erscheinen die Assyrer wie die Seleukiden als übermächtige Herren, denen Juda zu Steuern und Tributen verpflichtet ist. Wie die Assyrer treten auch die Seleukiden mit einem theologischen Anspruch und einem eigenen religiösen System auf. Wie 701 fühlen sich die Jerusalemer dem Heer der anrückenden Truppen hoffnungslos unterlegen und so wundert es nicht, dass sie sich in dieser Situation an die 
lich von den Epiphanieerzählungen zu unterscheiden, zumal im ganzen Zweiten Makkabäerbuch an keiner Stelle die in den Epiphanien auftretenden Figuren als "Engel“ (’ $\gamma \gamma \in \lambda \circ \varsigma)$ bezeichnet werden. ${ }^{85}$ Dennoch fungiert das Erzählen der Rettung von Sanherib als „Ersatz“ für eine Epiphanie, die eine epiphanieähnliche Wirkung erzielt: Nachdem Judas die Hilfe Gottes an Beispielen aus der Geschichte erinnert hat, wählt Judas, nach einem Blick in die Heilige Schrift, die Parole "Gottes

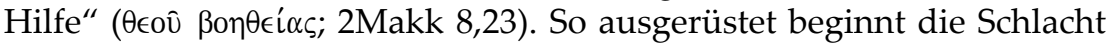
und die Truppen von Judas können tatsächlich siegen. Auf diese Weise zeigt sich in diesem Sieg die rettende Hilfe Gottes (vgl. тoû кupíov

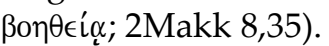

\subsection{Die vierte Epiphanieschilderung: Ein neuer Anführer (2Makk 11,8)}

Im elften Kapitel wird erneut eine militärische Auseinandersetzung beschrieben, die Judas Makkabäus gegen Lysias zu bestehen hat. ${ }^{86} \mathrm{Als}$ Judas und seine Leute bewaffnet in die Nähe des Gegners kommen,

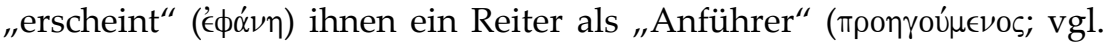
2Makk 10,29), der an ihrer Spitze kämpft. Der erschienene "Anführer" hat golden glänzende Waffen und ist in ein weißes Gewand gekleidet

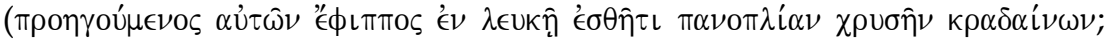
2Makk 11,8). ${ }^{87}$ Darüber hinaus bestärken „himmlischen Bundesgenos-

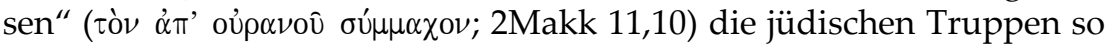
sehr, dass sie wie die Löwen erfolgreich gegen den Gegner kämpfen (2Makk 11,9-12). Explizit wird dabei betont, dass der Anführer als eine vom „barmherzigen Herrn vom Himmel“ gesandte Erscheinung inter-

Ereignisse aus der Zeit Hiskijas erinnert fühlen und Antiochus mit Sanherib vergleichen, die beide jeweils ihre Stellvertreter, Nikanor bzw. die assyrischen Gesandten, nach Jerusalem schicken.

85 Das Wort kommt im Zweiten Makkabäerbuch nur in Erinnerung an den Engel zur Zeit Sanheribs vor (2Makk 8,20; 15,22) oder in der Bitte an Gott um die Sendung eines „Engels“ (2Makk 11,6; 15,23). Daher rechtfertigt das Zweite Makkabäerbuch keine Identifizierung der in den Epiphanien auftretenden Gestalten mit „Engeln“, wie dies in der Literatur aber selbstverständlich zu lesen ist. Vgl. z.B. durchgehend bei BÉNEVOT, Makkabäerbücher, 40-41.184-185 oder bei GOLDSTEIN, II Maccabees, 213; vgl. auch die bei FISCHER, Heliodor, 125 und in Fußnote 29 genannten Belege.

86 Dieser beabsichtigt, in Jerusalem Griechen anzusiedeln, Gelder vom Tempel einzutreiben und das Hohepriesteramt zu verkaufen. Deshalb zieht er mit einer schlagkräftigen Truppe gegen Jerusalem und belagert die befestigten Plätze in Bethsur in der Nähe von Jerusalem (2Makk 11,1-3.4-5). Dass er dabei Gottes Macht (

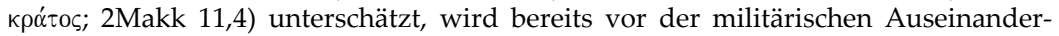
setzung betont.

87 2Makk 11,8: „Als sie aber dort bei Jerusalem waren, erschien ihnen als Führer ein Berittener in weißem Gewand und goldene Waffen schwingend." 


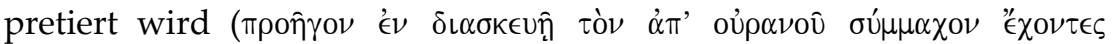

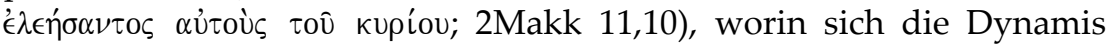

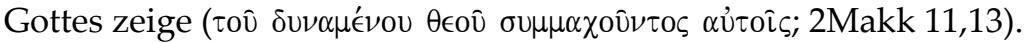

Wie bereits bei der vorangegangenen wird auch die vierte himmlische Erscheinung im elften Kapitel nicht explizit als „Epiphanie“ be-

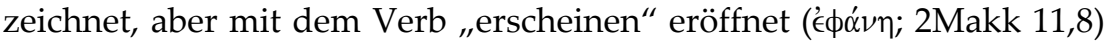
und vereint die für eine Epiphanie im Zweiten Makkabäerbuch typischen Merkmale (Pferd, Gold, prachtvolle Ausstattung etc.).

\subsection{Die fünfte Epiphanieschilderung: Eine Epiphanie des "alles Überschauenden“ (2Makk 12,22)}

Im Gegensatz zu den beiden vorangegangenen Epiphanieschilderungen wird die Erscheinung in 2Makk 12,22 explizit als Epiphanie

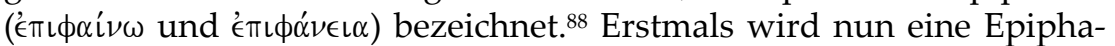
nie geschildert, bei der nicht erzählt wird, was gesehen wird. ${ }^{99}$ Aber es wird explizit betont, dass die Epiphanie göttlichen Ursprungs sei. Gott

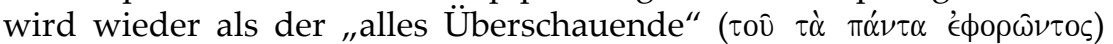
bezeichnet. ${ }^{90}$ Zudem wird betont, dass die Adressaten dieser Epiphanie

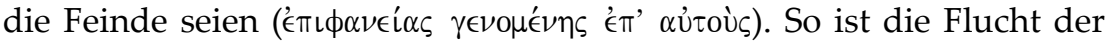

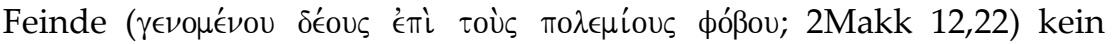

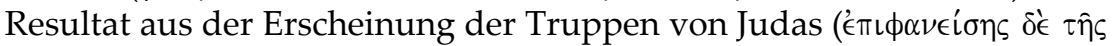

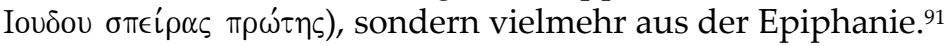

\subsection{Die sechste Epiphanieschilderung: Onias und Jeremia und die Epiphanie Gottes (2Makk 15,11-16.25-36)}

Wie die erste Epiphanie, von der gleich zu Beginn der Erzählung in 2Makk 3 berichtet wird, wird auch das letzte Kapitel der Erzählung durch eine Epiphanieschilderung beendet. Wie die erste ist auch diese

88 2Makk 12,22: „Als aber die erste Kompanie des Judas erschien und Furcht die Feinde befiel und Schrecken über sie kam infolge der Epiphanie dessen, der alles überschaut, wandten sie sich zu Flucht, der eine hierhin, der andere dorthin ..."

89 Die Vermutung, dass es sich hier in Analogie zu 2Makk 9,5 um himmlische Reiter gehandelt hat, ist reine Spekulation, vgl. DOBBELER, 1/2 Makk, 231; ähnlich DOMMERSHAUSEN, 1 Makkabäer, 162.

90 Eine ähnliche Gottesbezeichnung findet sich im Zweiten Makkabäerbuch nur noch

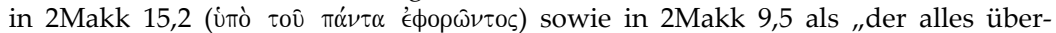

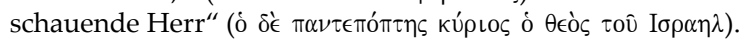

91 GolDSTEIN vermutet, dass es sich bei den Zahlen um eine Bezugnahme auf Lev 26,8 handelt, GOLDSTEIN, II Maccabees, 442. 
als eine doppelte Epiphanieschilderung gestaltet. In beiden tritt zudem der Hohepriester Onias auf (2Makk 15,11-16.25-36). 92

Judas und seine Leute müssen mit einem großen Angriff Nikanors rechnen. ${ }^{93}$ Daher ermutigt Judas seine Mitstreiter mit drei verschiedenen Maßnahmen, nicht furchtsam zu sein, sondern ihre Hoffnung auf

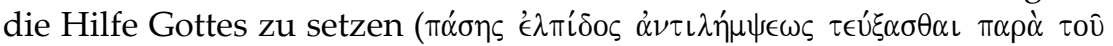
кupíov; 2Makk 15,7). ${ }^{94}$ Sie mögen auch jetzt auf die Hilfe vom Himmel

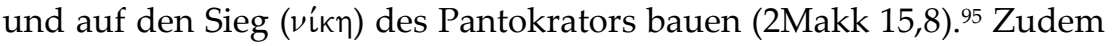
erinnert Judas seine Mitstreiter an die in Gesetz und Propheten überlieferten bestandenen Schlachten in der Geschichte (2Makk 15,9) sowie an den Vertragsbruch der Völker (2Makk 15,10). Schließlich erzählt er ihnen „einen glaubwürdigen Traum, eine Art Vision“96

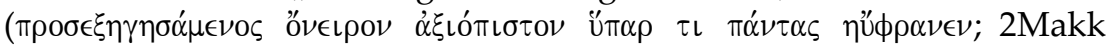
$15,11)$, die er gehabt habe $(\theta \in \omega \rho i \alpha ; 2$ Makk 15,12). In seinem Traum sei der Hohepriester Onias aufgetreten, dessen Charaktereigenschaften sehr gelobt werden (2Makk 15,12). ${ }^{97}$ Neben Onias ",erscheint" ( $€ \pi\llcorner\phi \alpha i \nu \omega)$ auch ein zweiter Mann, ein alter, ergrauter Herr. Dieser sei ebenfalls von herrlicher Gestalt gewesen und von ihm sei Glanz ausgegangen

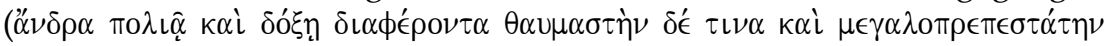

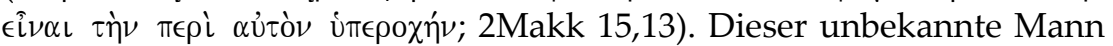
sei von Onias als „bruderliebender Mann“ (ó $\phi \iota \lambda \alpha \dot{\alpha} \delta \in \lambda \phi o \varsigma)$ und als der Prophet Jeremia vorgestellt worden (2Makk 15,14). ${ }^{98}$ Dass ausgerechnet Onias und Jeremia im Traum von Judas auftreten, könnte damit zusammenhängen, dass sie als exemplarische Repräsentanten der Schrift für "Gesetz und Propheten“ stehen könnten, und so im Traum die zu-

92 Zudem findet sich nur in 2Makk 3,24 und 15,27 die Kombination aus Epiphanie

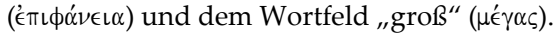

93 Diskutiert wird, ob 2Makk diesen Nikanor mit dem Nikanor aus 2Makk 8 identifiziert, vgl. hierzu TEDESCHE/ZEITLIN, second Book of Maccabees, 239. Vgl. zu 2Makk 15 auch HENTEN, Ideology, 265-282.

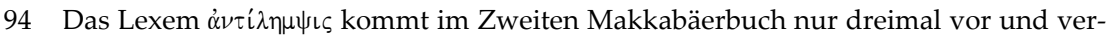
netzt die beiden Sanherib-Stellen miteinander (2Makk 8,19; 15,7 vgl. 11,26).

95 Dies könnte an die Siege in 2Makk 10,28 und 12,22-23 erinnern, die jeweils durch Epiphanien begleitet werden.

96 Zur Lesart vgl. HABICHT, 2. Makkabäerbuch, 277; ABEL, Les livres, 473

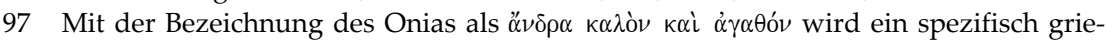
chischer Ausdruck verwendet, um einen vollkommenen Mann zu bezeichnen. Dies nimmt die Charakterisierung von Onias aus 2Makk 4,37 auf, wo dieser als $\sigma \omega \phi \rho o \sigma u ́ \nu \eta$ und $\epsilon \dot{\tau} \tau \alpha \xi^{\prime} \alpha$ und damit als ein "compleat Hellenistic gentleman" beschrieben wird (SCHWARTZ, 2 Maccabees, 239).

$98 \mathrm{Ob}$ die Nennung von Jeremia an dieser Stelle die Gestaltung des Ersten Festbriefes, in dem Jeremia ebenfalls auftritt (2Makk 2,1-8) beeinflusst hat, oder ob dies eher als ein Indiz zu werten ist, die Festbriefe als integralen Bestandteil der Komposition zu erachten, kann an dieser Stelle nicht entschieden werden. 
vor von Judas herangezogene biblische Bestärkung personifizieren. ${ }^{99}$

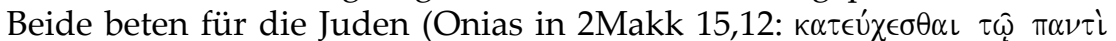

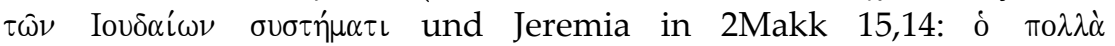

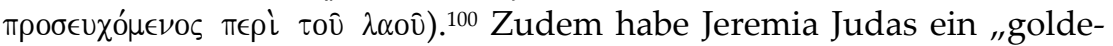

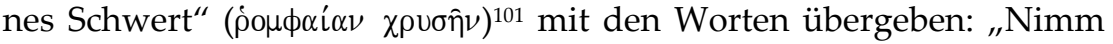
das heilige Schwert als Geschenk Gottes; mit ihm wirst du die Feinde

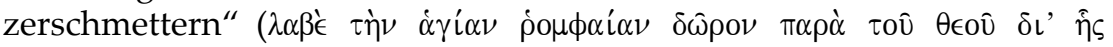

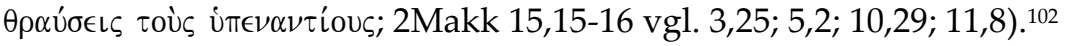

Im zweiten Teil (2Makk 15,20-29) folgt nun die Schilderung der Schlacht, zu deren Beginn Judas die Arme zum Himmel hochhebt und

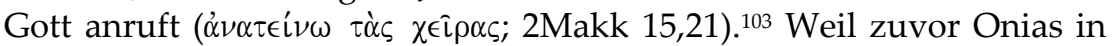

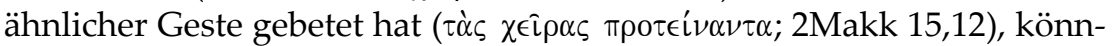
te durch diese Verbindung angedeutet werden, dass Judas in Kontinuität zu der aus makkabäischer Perspektive einzig rechtmäßigen kultischen Tradition am Jerusalemer Tempel steht. Mit erhobenen Händen

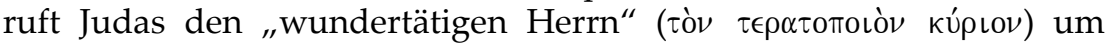
einen Sieg an (2Makk 15,21). Damit steht das militärisch unterlegene Heer in einer dreifachen Gebetsunterstützung: durch das Gebet des rechtschaffenen toten Hohenpriesters Onias, das des toten Propheten Jeremia und jenes des anwesenden militärischen Anführers Judas. In seinem Gebet erinnert Judas Gott an die Rettung Jerusalems zur Zeit

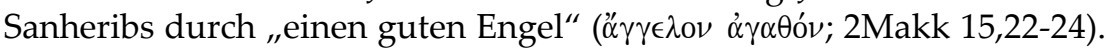
Auf diese Weise wird die Erzählung von 2Makk 15 mit 2Makk 8 ver-

99 Vgl. auch die Überlegungen, dass Onias und Jeremia hier als zwei Märtyrer gesehen werden, DOBBELER, 1/2 Makk, 246.

100 Dass Tote für Lebende beten, ist ein ungewöhnlicher und an dieser Stelle erstmals geäußerter Gedanke. In 2Makk 12,43-44 wird der Gedanke geäußert, dass man für die Toten beten solle. Diese Stellen seien der Grund für Luther gewesen, das Zweite Makkabäerbuch aus dem Kanon zu streichen, so BÉNEVOT, Makkabäerbücher, 245.

101 Dieses Motiv der Übergabe einer Waffe an den von Gott erwählten Führer des Kreuzfahrerheeres wird auch in einer sehr frühen Darstellung des Ersten Kreuzzugs von Raymond d'Aguilers verwendet, der explizit auf die Makkabäer verweist und immer wieder Schlachten schildert, die durch Epiphanien entschieden werden, vgl. hierzu auch AUFFARTH, Wege, 125-128.131-132. Für den Hinweis danke ich Hubertus Lutterbach.

102 Vgl. hierzu das Motiv des Schwerts im Jeremiabuch, v.a. Jer 27,35-37 [LXX] an der allerdings das Lexem $\mu \alpha \dot{\alpha} \alpha\llcorner\rho \alpha$ statt $\dot{\rho} о \mu \phi \alpha i \alpha$ verwendet wird: "and he will sharpen a sword against the Chaldeans, and against the inhabitants of Babylon, and upon her nobles and upon her wise men; a sword upon her warriors, and they shall be weakened: a sword upon their horses, and upon their chariots: a sword upon their warriors and upon the mixed people in the midst of her; and they shall be as women: a sword upon the treasures, and they shall be scattered upon her water" (Übersetzung der LXX nach BRENTON).

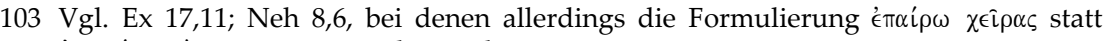
$\dot{\alpha} \nu \alpha \tau \in \dot{L} \nu \omega \tau \grave{\alpha} \varsigma \chi \in \hat{\imath} \rho \alpha \varsigma$ verwendet wird. 
netzt, in der auffallenderweise eine Epiphanie "fehlte", die durch die Erinnerung an die Belagerung Jerusalems durch Sanherib als „literarische Epiphanie“" „ersetzt" worden ist (vgl. ähnlich 2Makk 12,15). Unter Anrufung Gottes (so 2Makk 15,26-27) kämpfen die Leute von Judas gegen das Heer Nikanors und töten mehr als 35.000 Mann. Dabei gleicht die militärische Auseinandersetzung eher einer Liturgie denn Kampfhandlungen. ${ }^{104}$ Dieser Erfolg wird als „Epiphanie Gottes" be-

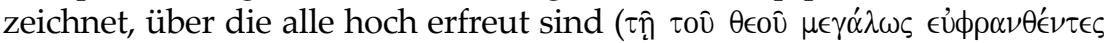
$\epsilon \pi \iota \phi \alpha \nu \in \dot{L} \alpha ; 2$ Makk 15,27). Als sie Nikanor unter den Toten finden (2Makk 15,29), wird dies als Ergebnis des "sichtbar gewordenen, epiphanen

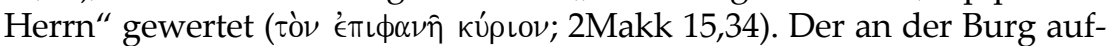
gehängte Kopf von Nikanor wird damit zum „sichtbaren Zeichen für die

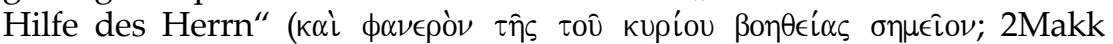
15,35; vgl. Jdt 13,15; 14,1 sowie 2Sam 17,54). Damit realisiert sich die in 2Makk 14,15 erflehte und in der Geschichte bereits erfahrene „Epiphanie“" Gottes ( $\mu \epsilon \tau$ ' ‘ $\pi\llcorner\phi \alpha \nu \epsilon i \alpha \varsigma)$. Das Leitmotiv in 2Makk 15, die

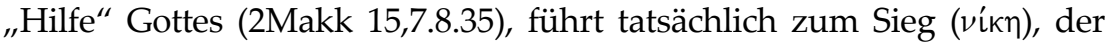
aus eigener militärischer Stärke nicht zustande gekommen wäre.

\subsection{Fazit zu „Epiphanie” im Zweiten Makkabäerbuch}

\begin{tabular}{|c|c|c|c|c|}
\hline & $\begin{array}{l}\text { Woher kom- } \\
\text { men die Epi- } \\
\text { phanien } \\
\text { (Herkunft) }\end{array}$ & $\begin{array}{l}\text { Wer sieht? } \\
\text { (Empfänger) }\end{array}$ & $\begin{array}{l}\text { Was wird gesehen? } \\
\text { (Gegenstand) }\end{array}$ & $\begin{array}{l}\text { Was sind die } \\
\text { Folgen des Ge- } \\
\text { sehenen? } \\
\text { (Folgen) }\end{array}$ \\
\hline $\begin{array}{l}\text { Vorwort } \\
(2,22)\end{array}$ & Himmlisch & $\begin{array}{l}\text { Die für das Ju- } \\
\text { dentum Käm- } \\
\text { pfenden }\end{array}$ & - & $\begin{array}{l}\text { (Wiederein- } \\
\text { weihung des } \\
\text { Tempels) }\end{array}$ \\
\hline $\begin{array}{l}\text { 1. Epiphanie } \\
(3,24-26 \\
3,31-34)\end{array}$ & Gott & $\begin{array}{l}\text { Heliodor } \\
\text { und Beglei- } \\
\text { ter }\end{array}$ & $\begin{array}{l}\text { 1. Pferd in präch- } \\
\text { tigem Geschirr } \\
\text { und Reiter in } \\
\text { Rüstung aus } \\
\text { Gold } \rightarrow \text { stürmen } \\
\text { auf Heliodor ein } \\
\text { zwei junge Män- } \\
\text { ner in kraftvoller } \\
\text { Schönheit, herr- } \\
\text { lich gekleidet } \\
\rightarrow \text { schlagen auf } \\
\text { Heliodor ein }\end{array}$ & $\begin{array}{l}\text { Heliodor: } \\
\text { Ohnmacht, } \\
\text { schwarz vor } \\
\text { Augen }\end{array}$ \\
\hline
\end{tabular}

104 So auch DOMMERSHAUSEN,1 Makkabäer, 179. 


\begin{tabular}{|c|c|c|c|c|}
\hline & & & $\begin{array}{l}\text { 2. zwei junge Män- } \\
\text { ner }\end{array}$ & $\begin{array}{l}\text { Gottes vor } \\
\text { Antiochus }\end{array}$ \\
\hline $\begin{array}{l}\text { 2. Epiphanie } \\
(5,1-4)\end{array}$ & $?$ & $\begin{array}{l}? \\
\text { (in der Stadt) }\end{array}$ & $\begin{array}{l}\text { großes Heer mit } \\
\text { Pferden, Reitern, } \\
\text { mit verschiedenen } \\
\text { Waffen ausgestat- } \\
\text { tete Soldaten in } \\
\text { Schlachtordnun- } \\
\text { gen, die durch die } \\
\text { Luft sprengen }\end{array}$ & $?$ \\
\hline $\begin{array}{l}\text { 3. Epiphanie } \\
(10,29-30)\end{array}$ & $\begin{array}{l}\text { vom Him- } \\
\text { mel }\end{array}$ & Feinde & $\begin{array}{l}\text { fünf prächtig ge- } \\
\text { kleidete Männer } \\
\text { auf Pferden mit } \\
\text { goldenen Zügeln } \\
\text { als Anführer } \\
\rightarrow \text { schleudern } \\
\text { Blitze und Pfeile }\end{array}$ & $\begin{array}{l}\text { Flucht der } \\
\text { Feinde, } \\
\text { Blindheit, } \\
\text { Verwirrung } \\
\rightarrow \text { Sieg der } \\
\text { jüdischen } \\
\text { Truppen, } \\
\text { viele Tote } \\
\end{array}$ \\
\hline $\begin{array}{l}\text { 4. Epiphanie } \\
(11,8)\end{array}$ & $\begin{array}{l}\text { Himmlische } \\
\text { Bundesge- } \\
\text { nossen }\end{array}$ & Feinde & $\begin{array}{l}\text { ein Reiter mit gol- } \\
\text { den glänzenden } \\
\text { Waffen und } \\
\text { einem weißen Ge- } \\
\text { wand als Anfüh- } \\
\text { rer } \rightarrow \text { schwingt } \\
\text { Waffen }\end{array}$ & $\begin{array}{l}\text { viele Tote, } \\
\text { Flucht der } \\
\text { Feinde } \\
\rightarrow \text { Sieg der } \\
\text { jüdischen } \\
\text { Truppen }\end{array}$ \\
\hline $\begin{array}{l}\text { 5. Epiphanie } \\
(12,22)\end{array}$ & $\begin{array}{l}\text { Der alles } \\
\text { Über- } \\
\text { schauende }\end{array}$ & Feinde & - & $\begin{array}{l}\text { Furcht, } \\
\text { Schrecken } \\
\text { und Flucht } \\
\text { der Feinde }\end{array}$ \\
\hline $\begin{array}{l}\text { 6. Epiphanie } \\
(15,11-16 ; \\
15,25-36)\end{array}$ & $\begin{array}{l}\text { Traum von } \\
\text { Judas } \\
\text { Gebet von } \\
\text { Judas }\end{array}$ & Judas & $\begin{array}{l}\text { 1. Hohepriester } \\
\text { Onias und Jere- } \\
\text { mia } \\
\rightarrow \text { überreicht } \\
\text { Judas ein golde- } \\
\text { nes Schwert } \\
\text { 2. in Gebet: Erin- } \\
\text { nerung an Ret- } \\
\text { tung vor Sanherib }\end{array}$ & $\begin{array}{l}\text { Sieg der jü- } \\
\text { dischen } \\
\text { Truppen }\end{array}$ \\
\hline
\end{tabular}


Blickt man auf alle Epiphanien unter den im Vorwort etablierten Kategorien, dann zeigen sich deutlich Unterschiede und Gemeinsamkeiten:

Erstens: Alle Epiphanien werden mit Blick auf ihre Herkunft in unterschiedlichen Graden explizit auf Gott zurückgeführt. Eine Ausnahme bildet nur die zweite Epiphanie (2Makk 5,1-4), deren Herkunft nicht deutlich wird. Bei den anderen jedoch fallen stets die typischen

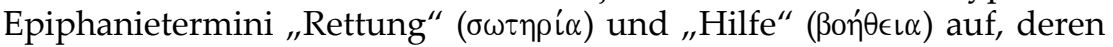

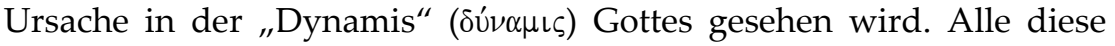
Epiphanien erzählen somit von der soteriologischen Qualität des Gottes Israel.

Zweitens: Anders als im Vorwort, das betont, dass die Epiphanien "den für die jüdische Sache voller Eifer und Tapferkeit Kämpfenden" (2Makk 2,21) zuteil werden, angekündigt, werden die Epiphanien in erster Linie von den Feinden gesehen: Es sind die Feinde, denen Gott sich in Epiphanien zeigt. Wie viel davon auch jeweils für die jüdischen Truppen sichtbar ist, bleibt meist offen. Auch hier bildet nur die zweite Epiphanie eine Ausnahme, die von denen "in der Stadt“ (2Makk 5,2) gesehen wird. Interessanterweise erfüllt nur diese Epiphanie, deren Deutung ambivalent bleibt, als Einzige die im Vorwort genannten Kriterien.

Drittens: Das, was in den Epiphanien jeweils gesehen wird, hat einen anderen Inhalt, sodass keine Epiphanie einer anderen gleicht. Dennoch kristallisiert sich ein gewisses Motivrepertoire heraus, das in unterschiedlichen Formen immer wieder variiert aufgegriffen wird: Es sind prächtige Pferde, Reiter und Männer, die jung, schön und kraftstrotzend sind, die über schlagkräftige Waffen, goldene Rüstungen und wertvolle Ausrüstung verfügen. Besonders die Farben Gold und Weiß werden immer wieder genannt. Beides sind Farben, die über eine besondere Leuchtkraft verfügen und glänzen, was die Grundbedeutung

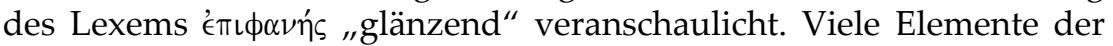
Epiphanieschilderungen im Zweiten Makkabäerbuch verweisen weniger auf biblische Tradition als vielmehr auf griechisch-hellenistische Epiphanieschilderungen. Dies zeigt, wie stark diese in der paganen Tradition verwurzelt sind. ${ }^{105}$

105 So z.B. das plötzliche und konkrete Auftreten von Gestalten aus der göttlichen Sphäre, die jedes Mal das Ziel haben, rettend gegen die Feinde schlachtentscheidend einzugreifen. So betont BICKERMANN, dass das Pferd der biblisch-jüdischen Tradition eher fremd sei und vielmehr hellenistisch-griechischen Vorstellungen entspreche, BICKERMANN, Héliodor, 7-40; wieder abgedruckt in erweiterter Fassung in: BICKERMANN, Studies, 176. 
Viertens: Alle Epiphanien - außer der zweiten - haben das gleiche Resultat: Blindheit, Ohnmacht und kopflose Flucht der Feinde sind die Folgen aus den Epiphanien, die jeweils zum Sieg der jüdischen Truppen und damit letztlich zur Wiedereinweihung des Jerusalemer Tempels führen, wie dies im Vorwort bereits vorwegnehmend angekündigt wird.

Vor diesem Hintergrund kann man eine deutlich abnehmende Dramatik der sechs Epiphanieschilderungen im Zweiten Makkabäerbuch erkennen: Während in der ersten die erscheinenden Pferde und Männer besonders ausgefeilt geschildert werden, nehmen die Anschaulichkeit und der Detailreichtum der Beschreibungen kontinuierlich ab. In der fünften Epiphanie wird sogar ganz auf eine Beschreibung dessen, was gesehen wurde, verzichtet (2Makk 12,22). Die sechste und letzte Epiphanie ist zudem insofern besonders, als dass Judas von einem Traum erzählt, in dem ihm Onias und Jeremia „erschienen“ seien (2Makk 15,13). Während die bisher in Epiphanien auftretenden Figuren nicht identifizierbar sind, handelt es sich bei diesen um zeitgenössische beziehungsweise literarisch bekannte Figuren (2Makk 15,22-24). Insofern kann man resümieren, dass die Epiphanien im Zweiten Makkabäerbuch immer literarischer werden und - man könnte vielleicht sogar sagen - zu „schriftgelehrter" Epiphanie werden. Dabei zeigen die letzten Epiphanien interessanterweise die gleiche Wirkung wie die ersten: Die jüdischen Truppen sind so bestärkt, dass sie Mut, Kraft und Stärke haben und die Feinde besiegen können. Diese Kampfkraft interpretie-

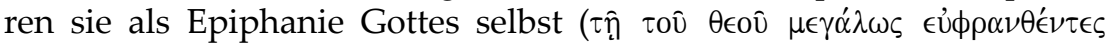
$\epsilon \pi\llcorner\phi \alpha \nu \in i$ a $\alpha 2$ Makk 15,27). So stehen am Ende der Erzählung keine Epiphanien mehr von prächtigen Reitern, blitzenden Waffen und jungen Männern in goldenen Gewändern, sondern „nur“ narrative Erinnerungen an Rettungssituationen, deren Erzählen so bestärkt, dass diese als Epiphanie Gottes selbst interpretiert werden. Im Erzählen dieser Epiphanien - so das Zweite Makkabäerbuch - zeige sich Gott selbst und bleibt doch unsichtbar. „Der unsichtbare Gott ,erscheint an Sich', das heißt, einfach so' und sinnlich gerade nicht wahrnehmbar - allein an seiner Wirkung gibt Er Sich zu erkennen und eben dadurch offenbart Er Sich auf Seine ,wunderbare' Weise! "106

Mit der Epiphanieschilderung wird somit ein Weg erzählt, mit dem Gott sich - gemäß der Tradition - als ein in der Geschichte handelnder Gott erweist, ohne aber in den Lauf der Welt anthropomorph einzugreifen. Zugleich kann man aber auch im Zweiten Makkabäerbuch eine 
sich steigernde Dynamik beobachten: Die Epiphanien, die am Anfang Erscheinungen sind, die von bestimmten Figuren wahrgenommen wurden, werden immer mehr zu Epiphanien Gottes selbst. So heißt es am Ende des Zweiten Makkabäerbuchs, dass der "Herr" selbst sichtbar erschienen sei und sich darin als der wahre kyrios epiphanes gezeigt

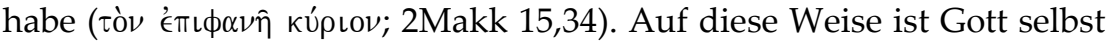
„epiphan" geworden. ${ }^{107}$

\section{Körperkonstruktionen in Auferstehung und Epiphanie im Zweiten Makkabäerbuch}

Beide Jenseitskonzepte - Auferstehung und Epiphanie - operieren mit Körperbildern: Auch wenn die Auferstehungstexte sehr zurückhaltend und vorsichtig mit Aussagen über das Leben nach dem Tod sind, so ist doch ersichtlich, dass sie sich das Leben als ein körperliches vorstellen: Nach dem Tod ist die Restitution des Körpers zu erwarten, wie in den beiden Reden der Mutter (2Makk 7,22-23.27-29), des dritten Sohnes (2Makk 7,11) sowie von Razi (2Makk 14,46), aber auch in dem Erscheinen des auferstandenen und körperlich identifizierbaren Onias (2Makk 15,12-16) deutlich wird. Dass nicht mehr über das Leben nach dem Tod erzählt wird, ist adäquat angesichts dessen, was Menschen über das Leben jenseits des Todes überhaupt aussagen können.

Anders verhält es sich bei den Epiphanieschilderungen: Alle epiphanen Gestalten haben einen Körper, der jeweils recht intensiv beschrieben wird. Dass dies nicht zwangsläufig so hätte sein müssen, zeigt allein die Erinnerung an den nicht körperlich beschriebenen „Boten“ oder „Engel“ aus der Sanherib-Erzählung (2Makk 8,19; 15,22-24). Daher ist die Frage besonders interessant, wie die Körper dieser Figuren jeweils beschrieben werden.

Zunächst fällt auf, dass alle epiphanen Gestalten Männer sind: Sie

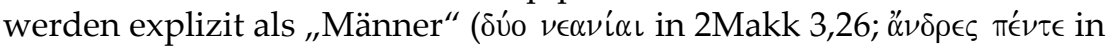

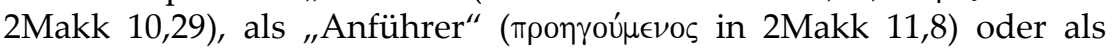

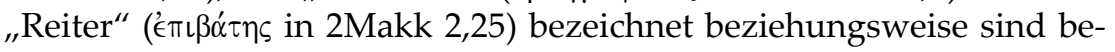
kannte Männer wie Onias und Jeremia.

Des Weiteren sind alle diese Männerfiguren von außerordentlicher Schönheit und Körperkraft. Die beiden jungen Männer aus der ersten Epiphanie werden beschrieben als Männer voller gewaltiger Kraft,

107 Dies ist etwas völlig anderes als von einem deus ex machina zu sprechen, der sich im Zweiten Makkabäerbuch zeige. Dieser Gott, der sich als der epiphane Gott erwiesen hat, wird visualisiert durch den an der Stadtmauer aufgehängten Kopf von Nikanor,

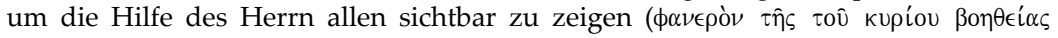

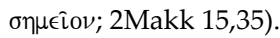




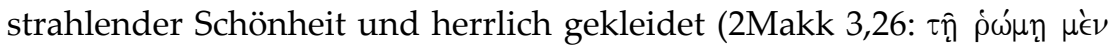

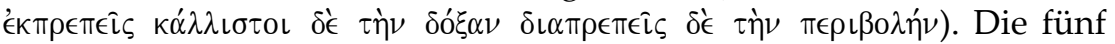
Männer, die in der dritten Epiphanie auftreten, sind prächtig gekleidete Männer auf Pferden mit goldenen Zügeln (2Makk 10,29). Der in der vierten Epiphanie auftretende "Anführer" trägt golden glänzende Waf-

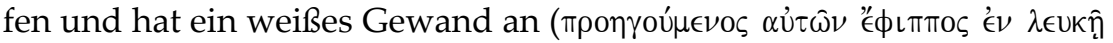

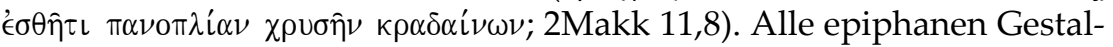
ten erscheinen so als junge, kraftvolle, schöne Männer.

Darüber hinaus sind alle epiphanen Gestalten sehr gute Soldaten, die mit bester militärischer Ausrüstung zielsicher und sehr erfolgreich kämpfen (vgl. 2Makk 5,2-4).

Die einzigen alten Männerfiguren sind Onias und Jeremia: Aber selbst Jeremia ist mit seinem weißen Haar eine glanzvolle Erscheinung mit bewundernswerter, großartiger Majestät (2Makk 15,13). Ebenso wird Onias als ein schöner und trefflicher Mann beschrieben $(\alpha \nu \delta \rho \alpha$

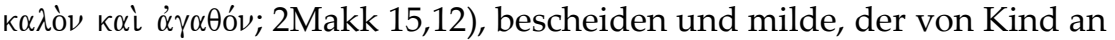

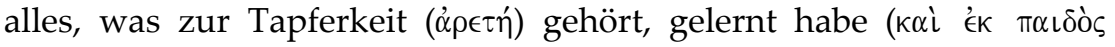

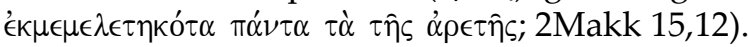

Durch die Epiphanien und die in ihnen auftretenden Gestalten wird zweierlei deutlich: Zum einen braucht Israel rettend eingreifende himmlische Gestalten, um die schlimmsten Bedrohungssituationen, Gefahren und militärischen Auseinandersetzungen zu bestehen. Dass Israel epiphane Unterstützung braucht, zeigt, dass man aus eigener militärischer Kraft allein die Auseinandersetzungen nicht hätte bestehen können. Darüber hinaus wird mit den epiphanen Gestalten ein bestimmtes Körper- und Männlichkeitsideal etabliert. Im Zweiten Makkabäerbuch sind es die epiphanen Gestalten, mit denen das Ideal eines kraftvollen, schönen und wehrhaften Mannes gezeichnet wird. Sie sind die Retter, die dem Volk in bedrängten Situationen machtvoll helfen können. Bei ihnen findet sich die Allianz zwischen einem kraftvollen Körper und der damit konnotierten politisch-militärischen Macht. Ein Spezifikum des Zweiten Makkabäerbuches ist es, dass dieses Ideal eben nicht von Menschen beziehungsweise Männern, sondern nur von himmlischen Wesen erfüllt wird.

Die Darstellung der in den Epiphanien präsentierten jungen, schönen Soldaten in goldenen Rüstungen und Waffen kann in vielfältiger Weise als ein Reflex auf die Lebensumwelt verstanden werden. So spiegeln die epiphanen Gestalten den Auftritt des idealtypischen hellenistischen Königs, der sich erfolgreich kämpfend und siegreich kraftstrotzend in prächtiger Rüstung zeigte. Zudem erscheinen die epiphanen Gestalten wie die Teilnehmer der großartigen Umzüge und Feierlichkeiten, wie sie im Hellenismus üblich sind, um die Erfolge des Königs 
öffentlich zu feiern und den eigenen Reichtum und die militärische Macht zur Schau zu stellen. Antiochus IV. Epiphanes hat bei seinen Feiern alle bisher gekannten Maßstäbe gesprengt: Besonders die Feierlichkeiten mit Festumzug und die dreißigtägige Feier 166 v. Chr. in Daphne, einem Vorort von Antiochia, haben an Pracht und Ausmaß alle bisherigen Feiern überboten. Vor seinem gesamten Reich und der ganzen griechischen Welt, aus der bis zu 300 Festgesandtschaften angereist waren, präsentierte Antiochus im Festumzug zuerst das Heer mit über 45.000 Soldaten unterschiedlichster Art und Waffengattung, die alle bestens ausgerüstet waren. Besonders die goldenen und golden glänzenden Rüstungen, Waffen und entsprechender Schmuck werden immer wieder erwähnt. Nach der Parade der 36 Kriegselefanten folgte der nicht-militärische Teil, bei dem auch wieder die Farbe Gold dominierte. 108

Darüber hinaus kann man in der Darstellung der epiphanen Gestalten eine kritische Auseinandersetzung mit dem griechisch-athletischen Körperideal sehen. ${ }^{109}$ Mit dem Ideal des schönen, durchtrainierten Athleten haben sich auch die Römer sehr kritisch auseinandergesetzt. Die römische Kritik traf vor allem die Nacktheit der Sportler und die daraus resultierenden Gefahren für die Sittlichkeit und das sinnlose Übermaß antrainierter Muskelkraft ohne praktischen Nutzen. ${ }^{110}$

Dass sich gerade im Zweiten Makkabäerbuch eine Auseinandersetzung mit dem griechischen Körperideal findet, ist keineswegs zufällig, schließlich hatte sich ja der Streit, der zum Makkabäeraufstand geführt hat, auch an der Errichtung eines Gymnasions und einer Ephebie in Jerusalem entzündet (vgl. 2Makk 4,9.12.14). ${ }^{111}$ Das Besondere an der Auseinandersetzung mit dem griechisch-athletischen Körperideal im Zweiten Makkabäerbuch ist, dass das Motiv des idealen, starken und unbesiegbaren Körpers aufgenommen wird, jedoch nicht in Gestalt von Menschen. Es wird in Epiphanien realisiert. Damit wird dieses Körperideal in eine himmlische Sphäre transzendiert.

108 Pol. 30.25.1/31.3 = Athen. 5,194; vgl. hierzu MitTAG, Antiochus, 282-295.

109 Im Zweiten Makkabäerbuch wird mit den epiphanen Gestalten eine spezielle Form von Männlichkeit konstruiert. Vgl. zur kritischen Männerforschung BauSteine Männer, Männerforschung; Meuser, Geschlecht; CONNELL/CONNELL, Mann; DöGE/ MEUSER, Männlichkeit; BOURDIEU, Herrschaft; MARTSCHUKAT/STIEGLITZ, Junge; CONNELL/CONNELL/MESSERSCHMIDT, Masculinity, 829-859; WACKER/RIEGER-GOERTZ, Mannsbilder. Vgl. hierzu auch DECKER, Sport.

110 Vgl. Plut. Cato Mai. 20,8; Cic. Tusc. 4,33, 70; Cic. off. 1,35, 126-127.129; 2, 17, 40; Tac. ann. 14,20 etc; vgl. hierzu MÄHL, Gymnastik, 40-54.

111 BOLYKI, Signal, 131-140. 
Welche Ideale etabliert die Erzählung für ihre „Helden“? Diese verdichten sich in zwei Motiven: erstens das Ideal der "Männlichkeit". ${ }^{112}$ Es wird im Vorwort (2Makk 2,21) im Kontext der Ankündigung der Epiphanien genannt, die denen zuteil werden, „die eifrig für das Juden-

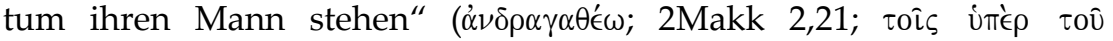

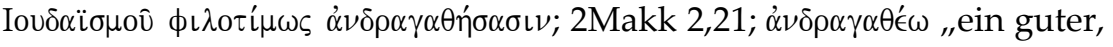
tapferer Mann sein"). ${ }^{113}$ Das Ideal der Männlichkeit durchzieht dann die Erzählung in positiver wie negativer Abgrenzung. Dieses im Vorwort genannte Ideal der Männlichkeit wird im Zweiten Makkabäerbuch nur ein einziges Mal für die Männer des Judas verwendet (2Makk 2,21; Substantiv in 14,18 $\left.\dot{\alpha} \nu \delta \rho \alpha \gamma \alpha \theta^{\prime} \dot{\alpha} \alpha\right)$. ${ }^{114}$ Interessanterweise findet sich somit die im Vorwort etablierte Kategorie in der weiteren Erzählung nicht mehr. Daher kann man von einer "Männlichkeitslücke“ in der Erzählung sprechen: Zwar wird vereinzelt vom Mut der jüdischen Truppen, ihrer militärischen Anführer oder Einzelner gesprochen (vgl.

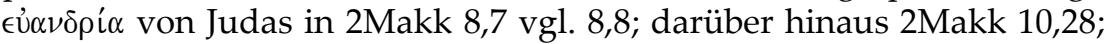
15,9.10). ${ }^{115}$, zugleich wird aber immer wieder deutlich gemacht, dass die Sieghaftigkeit der jüdischen Truppen nicht von ihnen oder ihren Anführern abhängt, sondern von Gott. ${ }^{116}$ So erläutert Judas in seiner geschichtstheologischen Rede (vgl. 2Makk 8,16-23) angesichts der Bedro-

112 Zum antiken Männlichkeitsideal vgl. GLEASON, Men; PORTER, Constructions; FISHER, Violence, 68-97; FOXHALL/SALMON, Men; ALSTON, Arms, 205-223; ROY, Masculinity, 111-135.

Zur kritischen Männerforschung im AT, die sich besonders mit bestimmten Männlichkeitsidealen auseinandersetzt, vgl. WACKER, Bibelwissenschaft, 126-131; Einzelstudien vgl. z.B. CLINES, David, 212-243; CLINES, He-Prophets, 311-328; HAESEN, Mose, 138-143.

Im Neuen Testament vgl. MoOre/CAPel ANDERSON, New Testament; Clines, Ecce vir, 352-375; LEUTZSCH, Konstruktionen, 600-618.

113 Dieses Verb findet sich auffallenderweise bis auf Est 10,21 ausschließlich im Kontext der Makkabäerüberlieferungen.

114 Anders verhält es sich interessanterweise im Ersten Makkabäerbuch, wo es breite Verwendung findet und mit ihm von den Erfolgen der makkabäischen Truppen berichtet wird (1Makk 5,56.61.67; 8,2; 9,22; 10,15; 16,23; 4Makk 1,8). Damit unterscheidet sich das Zweite Makkabäerbuch grundlegend vom Ersten.

115 Eine Beschreibung von Judas oder gar seiner Statur findet sich nicht. Vielmehr scheint Judas auch schutzbedürftig zu sein, wie durch den flankierenden Schutz von zwei der fünf epiphanen Gestalten in der dritten Epiphanieschilderung deutlich wird (2Makk 10,29-30).

116 Dies scheint nicht nur traditionsgeschichtlich mit den JHWH-Kriegsvorstellungen als vielmehr mit der Übertragung des hellenistischen Königsideals auf den Gott Israels zusammenzuhängen. Wie sich der hellenistische König in erster Linie durch seinen Erfolg legitimiert, so zeigt sich das Gott- und König-Sein des Gottes Israels in seinem Beistand für die jüdischen Truppen und ihrer Sieghaftigkeit, vgl. hierzu SCHUBART, Königsideal, 1-26; HeUSS, Ursprung, 208-213; COBET, König, 11-66; GEHRKE, König, 247-277; STEWART, Faces; HERZ, Könige, 27-40. 
hung durch Nikanor, vor der viele in geradezu „unmännlicher“ Weise $(\delta \in\llcorner\lambda \alpha \nu \delta \rho \epsilon \omega ;$; 2 Makk 8,13) weggelaufen sind, dass Israel allein auf den allmächtigen Gott baue, der die Angreifer und die gesamte Welt mit einem Wink niederstrecken könne (2Makk 8,18). Im Gegensatz dazu entspricht eine andere Figur der Erzählung dem Ideal der Männlichkeit und des Mutes. Diese Figur ist - erstaunlicherweise - eine Frau: Die Mutter der sieben Söhne hat, so heißt es explizit in 2Makk 7,21, „männ-

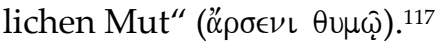

Das zweite Ideal, das in der Erzählung etabliert wird, erfüllt Onias beispielhaft: das Ideal der Tugend, der Tapferkeit, der $\alpha \rho \epsilon \tau \dot{~(2 M a k k ~}$ 15,16 vgl. 4,2). Es findet sich signifikanterweise nur bei Eleaser ( $\dot{\alpha} \rho \in \tau \dot{\eta} ;$ 2Makk 6,31; vgl. 6,18) und bei Razi (2Makk 14,37.38.42). ${ }^{118}$ Beide werden als tapfer und tüchtig beschrieben und entsprechen zugleich dem anderen Ideal der Männlichkeit: Sie sind von edler und männlicher Gesinnung (Eleaser: $\dot{\alpha} \nu \delta \rho \epsilon i \omega \varsigma ; 2 M a k k ~ 6,27$; Razi: $\dot{\alpha} \nu \delta \rho \omega \delta \omega \varsigma ;$ 2Makk 14,43). ${ }^{119}$

Auf den ersten Blick scheint somit im Zweiten Makkabäerbuch ein sehr militärisches und allein körperbetontes Ideal vorzuherrschen. Doch dieser erste Eindruck ändert sich, wenn man auf die Figuren der diesseitigen Textwelt des Zweiten Makkabäerbuches schaut: Hier sind es keineswegs die großen militärischen Anführer, die schön und kraftstrotzend sind. Im Gegenteil: Sie brauchen die Epiphanien zu ihrer Unterstützung. Vielmehr entsprechen dem Körperideal der Männlichkeit und der Tapferkeit andere Figuren: Dies sind Eleaser, die Mutter der sieben Söhne, Razi und Onias, aber auch Judas (Festhalten an der Tora, vgl. 2Makk 5,27). Auf diese Weise wird im Zweiten Makkabäerbuch nicht das Bild einer wehrhaften Nation aus sich heraus aufgebaut, sondern es wird betont, dass die eigentliche Kraft Israels im Halten der Tora und im Festhalten am Tempel liegt - auch und gerade dann, wenn es bis zum Äußersten geht. Die Helden des Zweiten Makkabäerbuches sind die Standhaften, die Schwachen und die zu Tode Gefolterten. ${ }^{120}$

\section{Auferstehung und Epiphanie im Zweiten Makkabäerbuch. Ein Vergleich}

Nach diesem Durchgang wird deutlich, dass Auferstehung und Epiphanie für die Erzählung zentrale Denkfiguren sind. Beide durch-

117 Vgl. hierzu auch TOMES, Heroism, 171-199.

118 So auch HABICHT, 2. Makkabäerbuch, 277.

119 Vgl. hierzu auch SCHWARTZ, 2 Maccabees, 51.

120 So auch SCHWARTZ: "Martyrs, rather than soldiers, are the real heroes of the book", SCHWARTZ, 2 Maccabees, 50. 
brechen aus unterschiedlichen Perspektiven die Grenze, die der Tod darstellt. Während der Gedanke an die Auferstehung den Figuren der Erzählung Hoffnung auf ein Leben nach dem Tod vermittelt, ragt die jenseitige Welt Gottes durch die in den Epiphanien auftretenden Gestalten in die diesseitige Welt hinein.

Angesichts dessen ist es interessant, zu fragen, ob und inwiefern die prospektiv im Vorwort genannten Charakteristika der Epiphanien (2Makk 2,21) auch auf die Auferstehungstexte zutreffen. Die erste Kategorie, die Herkunft, wird in umgekehrter Richtung erfüllt: Während es sich bei den Epiphanien um „vom Himmel kommende Epiphanien“

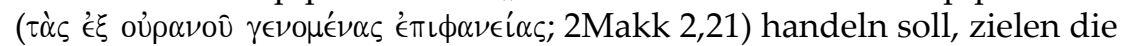
Auferstehungstexte umgekehrt auf ein jenseitiges Leben. Die zweite Kategorie bezieht sich auf den Adressatenkreis: ${ }^{121}$ Die Epiphanien wer-

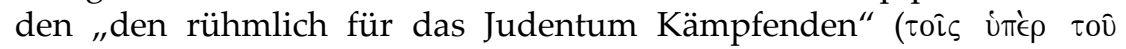

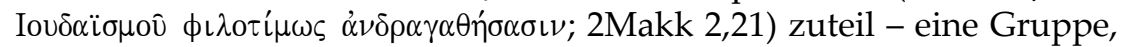
die mit den Märtyrern und den für das Judentum Kämpfenden, denen Auferstehung in Aussicht gestellt wird, weitgehend identisch ist. Die dritte Kategorie thematisiert das Ziel der Epiphanien, das in der Rückeroberung des Landes, dem Verjagen der Feinde, der Rückeroberung des Heiligtums, der Befreiung der Stadt und der Wiederaufrichtung der Gesetze besteht (2Makk 2,21-22). Diesem Ziel ist der Gedanke der Auferstehung verpflichtet: So erleiden die Söhne und ihre Mutter sowie Razi $(2$ Makk 14,46) den Märytrertod, weil sie die Tora eben nicht zu brechen bereit sind. Die gefallenen Soldaten in 2Makk 12,43-45 haben für das Judentum gekämpft. Der auferstandene und für die kämpfenden jüdischen Truppen betende Onias (2Makk 15,12-16) steht als der letzte Hohepriester für den rechtmäßigen Kult im Tempel. ${ }^{122}$ Die vierte Kategorie benennt als theologische Begründung für die Epiphanien die

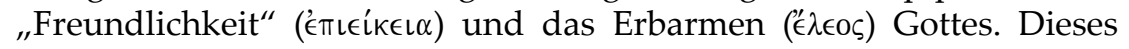
Wortfeld findet sich explizit in den Reden der Mutter (2Makk 7,23.29) als theologische Begründung für die Auferstehungshoffnung. Auf diese Weise zeigt sich, dass die von der Erzählung selbst etablierten Kategorien für die Epiphanien gerade auch von dem anderen Jenseitskonzept, der Auferstehung, erfüllt werden.

Beide Jenseitskonzepte, Auferstehung und Epiphanie, die sich wie zwei getrennte Fäden durch die Erzählung ziehen, kommen am Ende zusammen: Onias erscheint in der letzten Epiphanie Judas im Traum

121 Wie oben erläutert, sind die Epiphanien de facto auch und gerade für die Feinde zu sehen.

122 Onias trage zudem die Züge des Märtyrers Eleaser aus 2Makk 6, so KellERMANN, Auferstanden, 86. 
als Auferstandener. Damit vereint der letzte rechtmäßige Hohepriester beide Vorstellungen.

Sowohl in den Epiphanien als auch in den Auferstehungstexten wird zudem die Frage nach dem Körper thematisiert: So wird in den Auferstehungstexten deutlich, dass man sich das Leben nach dem Tod als ein körperliches vorstellt. Umgekehrt haben die epiphan auftretenden Gestalten einen Körper, der intensiv beschrieben wird.

Auferstehung und Epiphanie sind nicht verschiedene, sondern zwei komplementäre Denkfiguren, die im Zweiten Makkabäerbuch zusammen gehören, aufeinander bezogen sind und daher auch nicht auseinandergerissen werden dürfen. Im Zweiten Makkabäerbuch ist Auferstehung kein isolierbares Konzept. Vielmehr bilden Auferstehung und Epiphanie zusammen den Horizont der Jenseitsvorstellungen.

\section{Bibliographie}

ABEL, Félix-Marie, Les livres des Maccabées, Paris 1949.

ALSTON, Richard, Arms and the man: soldiers, Masculinity and power in Republican and Imperial Rome, in: Foxhall, Lin/Salmon, John (Hg.), When Men were Men. Masculinity, power and identity in classical antiquity, London/New York 1998, 205223.

ARENHOEVEL, Diego, Die Theokratie nach dem 1. und 2. Makkabäerbuch (WSAMA.T 3), Mainz 1967.

ARNOLD, Heinz Ludwig/SINEMUS, Volker (Hg.), Grundzüge der Literatur- und Sprachwissenschaft. Bd 1: Literaturwissenschaft, München ${ }^{5} 1978$.

AUfFARTH, Christoph, Irdische Wege und himmlischer Lohn. Kreuzzug, Jerusalem und Fegfeuer in religionswissenschaftlicher Perspektive, Göttingen 2002.

BARTLETT, John R., 2 Maccabees, in: Dunn, James D. G. (Hg.), Eerdmans Commentary on the Bible, Grand Rapids 2003, 831-850.

BARTLETT, John R., The first and second Book of Maccabees, Cambridge 1973.

BAUSTEINE Männer (Hg.), Kritische Männerforschung. Neue Ansätze in der Geschlechtertheorie, Berlin/Hamburg 1996.

BÉNEVOT, Hugo, Die beiden Makkabäerbücher, Bochum 1931.

BEYERLE, Stefan, Die Gottesvorstellung in der antik-jüdischen Apokalyptik (JSJ.S 103), Leiden/Boston 2002.

BICKERMANN, Elias, Héliodor au temple de Jérusalem, AIPh 1939-1944.

BICKERMANN, Elias, Studies in Jewish and Christian History, Bd. 2, Leiden 1980.

BIEBERSTEIN, Klaus, Der lange Weg zur Auferstehung der Toten. Eine Skizze zur Entstehung der Eschatologie im Alten Testament, in: Bieberstein, S./Kosch, D. (Hg.), Auferstehung hat einen Namen. Biblische Anstöße zum Christsein heute (FS HermannJosef Venetz), Luzern 1998, 3-16.

BOLYKI, János, "As soon as the Signal was given" (2 Macc 4:14). Gymnasia in the Service of Hellenism, in: Xeravits, G.G./Zsengellér, J. (Hg.), The Books of Maccabees. History, Theology, Ideology (JSJ.S 118), Leiden/Boston 2007, 131-140.

BOURDIEU, Pierre, Die männliche Herrschaft, Frankfurt a. M. 2005.

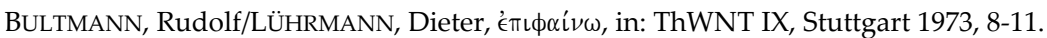


Clines, David J., David the Man. The Construction of Masculinity in the Hebrew Bible, in: id., Interested Parties: The Ideology of Writers and Readers of the Hebrew Bible (JSOT.S 205), Sheffield 1995, 212-243.

Clines, David J., Ecce vir Or Gendering the Son of Man, in: Exum, Cheryl/Moore, Stephen D. (Hg.), Biblical Studies/Cultural Studies (JSOT.S 266), Sheffield 1998, 352375.

ClinES, David J., He-Prophets: Masculinity as a Problem for the Hebrew Prophets and their Interpreters, in: Hunter, Alistair G./ Davies, Philip R. (Hg.), Sense and Sensitivity (FS R. Caroll) (JSOT.S 348), Sheffield 2002, 311-328.

COBET, Justus, König, Anführer, Herr, Monarch, Tyrann, in: Welskopf, Elisabeth Charlotte (Hg.), Soziale Typenbegriffe im alten Griechenland, Bd. 3, Berlin 1981, 1166.

ConNell, Robert W./ConNelL, Raewyn, Der gemachte Mann. Konstruktion und Krise von Männlichkeiten, Opladen 1999.

CONNELl, Robert W./CONNELl, Raewyn/MESSERSCHMIDT, James W., Hegemonic Masculinity. Rethinking the Concept, in: Gender \& Society 19 (2005) 829-859.

DECKER, Wolfgang, Sport in der griechischen Antike. Vom minoischen Wettkampf bis zu den Olympischen Spielen, München 1995.

DiBeliUS, Martin/CONZELMANN, Hans, Die Pastoralbriefe (HNT), Tübingen ${ }^{4} 1966$.

DOBBELER, Stephanie von, Die Bücher 1/2 Makk (NSK.AT 11), Stuttgart 1997.

DöGE, Peter/MEuSER, Michael (Hg.), Männlichkeit und soziale Ordnung. Neuere Beiträge zur Geschlechterforschung, Opladen 2001.

DOMMERSHAUSEN, W., 1 Makkabäer / 2 Makkabäer (NEB), Würzburg 1985.

DONELSON, Lewis R., Pseudepigraphy and ethical argument in the pastoral epistles, Tübingen 1986.

DORAN, R., Temple Propaganda. The Purpose and Character of 2 Macc (CBQ 12), Washington 1981.

ENGEL, Helmut, Die Bücher der Makkabäer, in: Zenger, Erich u.a. (Hg.), Einleitung in das Alte Testament, Stuttgart 62006.

ENERMALM-OGAWA, Agneta, Un langage de priere juif en grec. Le temoignage des deux premiers livres des Maccabees (CB.NT 17), Stockholm 1987.

FISCHER, Alexander Achilles, Tod und Jenseits im Alten Orient und Alten Testament, Neukirchen 2005.

FISCHER, Th., Heliodor im Tempel von Jerusalem. Ein hellenistischer Aspekt der "frommen Legende“, in: Herrmann, Siegfried (Hg.), Prophetie und geschichtliche Wirklichkeit im alten Israel (FS S. Herrmann), Stuttgart 1991, 121-138.

FISHER, Nick, Violence, masculinity and the law in classical Athens, in: Foxhall, Lin/Salmon, John (Hg.), When Men were Men. Masculinity, power and identity in classical antiquity, London/New York 1998, 68-97.

FOXHALL, Lin/SALMON, John (Hg.), Thinking Men. Masculinity and its SelfRepresentation in the Classical Tradition, London/New York 1998.

FRENSCHKOWSKI, Marco, Offenbarung und Epiphanie. Die verborgene Epiphanie in Spätantike und frühem Christentum, 2 Bde. (WUNT II/80), Tübingen 1995/1997.

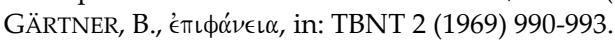

GEHRKE, Hans-Joachim, Der siegreiche König. Überlegungen zur hellenistischen Monarchie, in: AkuG 64 (1982) 247-277.

GINZBERG, Louis (Hg.), The Legends oft the Jews, Bd. VI, Philadelphia 1946.

GladiGOW, Burkhard/SCRIBA, Albert/LÜHRMANN, Dieter, Art. „Epiphanie“, in: RGG 2, Tübingen ${ }^{4} 1999,1367-1373$.

GleAsON, Maud W., Making Men. Sophists and Self-Presentation in Ancient Rome, Princeton 1995. 
GoldSTEIN, Jonathan A., II Maccabees (AncB 41A), New York 1984.

GRAF, Fritz, Epiphanie, in: Der Neue Pauly 3 (1997) 1150-1152.

GRIMM, Carl Ludwig Willibald, Das zweite, dritte und vierte Buch der Maccabäer, Leipzig 1857.

GUTTENBERGER, Gudrun, $\Omega \phi \theta \eta$. Der visuelle Gehalt der frühchristlichen Erscheinungstradition (Teil 1), in: BZ 52 (2008) 40-63.

GuTtENBERGER, Gudrun, $\Omega \phi \theta \eta$. Der visuelle Gehalt der frühchristlichen Erscheinungstradition und mögliche Folgerungen für die Entstehung und Entwicklung des frühchristlichen Glaubens an die Auferstehung Jesu (Teil 2), in: BZ 52 (2008) 161-173.

HABICHT, Christian, 2. Makkabäerbuch (JSHRZ 1,3), Gütersloh 1976.

HAESEn, Mareike, Mose der Mann. Konstruktion von Männlichkeit im Alten Testament, in: BiKi 63 (2008) 138-143.

HANHART, Robert, Zum Text des 2. und 3. Makkabäerbuches. Probleme der Überlieferung, der Auslegung und der Ausgabe, Göttingen 1961.

HASLER, Victor, Epiphanie und Christologie in den Pastoralbriefen, in: ThZ 33 (1977) 193209.

HENTEN, Jan Willem van, Royal Ideology: 1 and 2 Maccabees and Egypt, in: Rajak, Tessa (Hg.), Jewish Perspectives on Hellenistic Rulers (Hellenistic Culture and Society 50), Berkeley 2007, 265-282.

HERZ, Peter, Hellenistische Könige. Zwischen griechischen Vorstellungen vom Königtum und Vorstellungen ihrer einheimischen Untertan, in: Small, Alastair M. (Hg.), Subject and Ruler. The Cult of the Ruling Power in Classical Antiquity. Papers presented at a conference held in The University of Alberta on April 13-16, 1994, Ann Arbor 1976, 27-40.

Heuss, Alfred, Ursprung und Idee, in: Heuss, Alfred/Aymard, A., La monarchie hellénistique, in: Relazioni del X Congresso Internazionale di Szienze Storiche. Vol. II. Storia dell' Antichità, Firenze 1955, 208-213.

JEREMIAS, Jörg, Theophanie. Die Geschichte einer alttestamentlichen Gattung, Neukirchen-Vluyn ${ }^{2} 1977$.

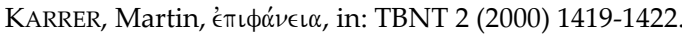

KellermanN, Ulrich, Auferstanden in den Himmel. 2 Makkabäer 7 und die Auferstehung der Märtyrer (SBS 95), Stuttgart 1979.

KellermanN, Ulrich, Das Gotteslob der Auferweckten. Motivgeschichtliche Beobachtungen in Texten des Alten Testaments, des frühen Judentums und Urchristentums (BThSt 46), Neukirchen-Vluyn 2001.

KELLERMANN, Ulrich, Überwindung des Todesgeschicks in der alttestamentlichen Frömmigkeit vor und neben dem Auferstehungsglauben, in: ZThK 73 (1976) 259-282.

LÄMMERT, Eberhard, Bauformen des Erzählens, Stuttgart ${ }^{8} 1989$.

LANG, Bernhard/MCDANNELL, Colleen, Der Himmel. Eine Kulturgeschichte des ewigen Lebens, Frankfurt 1996.

LEUTZSCH, Martin, Konstruktionen von Männlichkeit im Urchristentum, in: Crüsemann, Frank u.a. (Hg.), Dem Tod nicht glauben (FS Schottroff), Gütersloh 2004, 600-618.

LÜHRMANN, Dieter, Epiphaneia. Zur Bedeutungsgeschichte eines griechischen Wortes, in: Jeremias, Gert (Hg.), Tradition und Glaube. Das frühe Christentum in seiner Umwelt (FS K.G. Kuhn), Göttingen 1971, 185-199.

MÄHL, Eberhard, Gymnastik und Athletik im Denken der Römer, Amsterdam 1974.

MARIANO, Antonio, L'episodio di Eliodoro al tempio (2 Mac 3,1-40) nel contesto dello scontro tra e guidaismo, in: Sal 76 (2005) 421-456.

MARTINEZ, Matias/SCHEFFEL, Michael, Einführung in die Erzähltheorie, München 22000.

MARTSCHUKAT, Jürgen/STIEGLITZ, Olaf, Es ist ein Junge! Einführung in die Geschichte der Männlichkeiten in der Neuzeit, Tübingen 2005. 
MEUSER, Michael, Geschlecht und Männlichkeit. Soziologische Theorie und kulturelle Deutungsmuster, Opladen 1998.

MitTAG, Peter Franz, Antiochus IV. Epiphanes. Eine politische Biographie (Klio. Beihefte Neue Folge 11), Berlin 2006.

MOMigliano, Arnold, Alien Wisdom. The Limits of Hellenization, Cambridge 1978.

Moore, Stephen D./CAPEL ANDERSON, Janice (Hg.), New Testament Masculinities, Leiden/Bosten 2004.

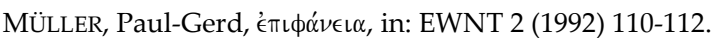

NICKELSBURG, George W.E., Resurrection, Immortality and Eternal Life in Intertestamental Judaism (HTS XXVI), Expanded Edition, Harvard 2006.

NICKLAS, Tobias, Aus erzählter Geschichte „lernen“. Eine narrative Analyse von 2 Makk 8, in: JSJ 32 (2001) 25-41.

NILSSON, Martin P., Geschichte der griechischen Religion, Bd. II, München ${ }^{2} 1961$.

OberLINNER, Lorenz, Die „Epiphaneia“ des Heilswillens Gottes in Christus Jesus. Zur Grundstruktur der Pastoralbriefe, in: ZNW 71 (1980) 192-312.

OBERLINNER, Lorenz, Epiphanie, in: NBL I, Zürich 1991, 552-554.

PASSOW, Friedrich, Handwörterbuch der griechischen Sprache, neu bearbeitet von Rost, V.Ch.F./Palm, F., Leipzig ${ }^{5} 1841$ (Nachdr. 2008).

PAX, Elpidius, Epiphaneia. Ein religionsgeschichtlicher Beitrag zur biblischen Theologie, München 1955.

PAX, Elpidius, Art. „Epiphanie“, in: RAC 5, Stuttgart 1962, 823-909.

PfEIFFER, Henrik, Jahwes Kommen von Süden. Jdc 5, Hab 3, Dtn 33 und Ps 68 in ihrem literatur- und theologiegeschichtlichen Umfeld (FRLANT 211), Göttingen 2005.

PFISTER, E., Art. „Epiphanie“, in: Pauly-Wissowa, Realencylopädie der classischen Altertumswissenschaften, Supp. 4 (1924) 277-323.

PORTER, James I., Constructions of the Classical Body, Michigan 1999.

PRITChETT, Kendrick William, The Greek state of War, Part III: Religion, Berkeley 1979.

RoLOFF, Jürgen, Der erste Brief an Timotheus (EKK 15), Zürich 1988.

RoY, Jim, The masculinity of the Hellenistic King, in: Foxhall, Lin/ Salmon, John (Hg.), When Men were Men. Masculinity, power and identity in classical antiquity, London/New York 1998, 111-135.

SCHMITZ, Barbara, Geschaffen aus dem Nichts? Die Funktion der Rede von der Schöpfung im Zweiten Makkabäerbuch, in: Nicklas, Tobias /Zamfir, Korinna (Hg.), Creation in Early Jewish and Christian Literature (DCLSt), Berlin et al., 2010 [forthcoming].

SCHUBERT, Kurt, Die Entwicklung der Auferstehungslehre von der nachexilischen Zeit bis zur frührabbinischen Zeit, in: BZ 6 (1962) 177-214.

SCHUBART, Wilhelm, Das hellenistische Königsideal nach Inschriften und Papyri, in: APF 12 (1937) 1-26.

SCHWARTZ, Daniel R., 2 Maccabees (CEJL), Berlin/New York 2008.

SCHWEIKLE, Günther, Art. „Episode“, in: Metzler Literatur Lexikon, Stuttgart 21990, 131.

SCRIBA, Albrecht, Die Geschichte des Motivkomplexes Theophanie. Seine Elemente, Einbindung in Geschehensabläufe und Verwendungsweisen in altisraelitischer, frühjüdischer und frühchristlicher Tradition (FRLANT 167), Göttingen 1995.

SETZER, Claudia, Resurrection of the Body in Early Judaism and Early Christianity. Doctrine, Community and Self-Definition, Boston/ Leiden 2004.

SÖDING, Thomas, Art. „Epiphanie“, in: LThK 3, Freiburg 31995, 719-720.

SPEYER, W., Die Hilfe und Epiphanie einer Gottheit, in: Dassmann, Ernst/Frank, K. Suso (Hg.), Pietas (FS Kötting), Münster 1980, 55-77.

STEWART, Andrew F., Faces of Power. Alexander's Image and Hellenistic Politics, Berkeley 1993. 
TeDESCHE, Sidney Saul/ZeITLIN, J. Solomon, The second Book of Maccabees (JAL), New York 1954.

DOMMERSHAUSEN, Werner, 1 Makkabäer / 2 Makkabäer (NEB), Würzburg 1985.

TOMES, Roger, Heroism in 1 and 2 Maccabees, in: Biblical Interpretation 15 (2007) 171-199.

VERSNEL, Hendrik S., What did Ancient Man see when He saw a God?, in: Plas, Dirk van der (Hg.), Effigies Dei, Leiden 1987, 42-55.

WACHSMUTH, D., Epiphanie, in: Der Kleine Pauly 5 (1975) 1598-1601.

WACKER, Marie-Theres, Bibelwissenschaft und Männerforschung, in: BiKi 63 (2008) 126131.

WACKER, Marie-Theres/RIEGER-GOERTZ, Stefanie, Mannsbilder. Kritische Männerforschung und Theologische Frauenforschung im Gespräch (Theologische Frauenforschung in Europa 21), Münster 2006.

WACKER, Marie-Theres, Theologie einer Mutter - eine Mutter als Theologin. Feministische-exegetische Anmerkungen zu 2 Makk 7, in: Riedel-Spangenberger, Ilona (Hg.), „Gott bin ich, kein Mann“. Beiträge zu einer Hermeneutik der biblischen Gottesrede (FS H. Schüngel-Straumann), Paderborn 2006, 259-270.

WERTHEIM, Ursula, Fabel und Episode in Dramatik und Epik, in: NDL 12 (1964) 87-107.

WeStermanN, Claus, Das Buch Jesaja. Kapitel 40-66 (ATD 19), Göttingen 1966.

WeStermanN, Claus, Lob und Klage in den Psalmen, Göttingen 51977.

WILLIAMS, David S., Recent Research in 2 Maccabees, in: Currents in Biblical Research 2 (2003) 69-83.

WILPERT, Gero von, Episode, in: id., Sachwörterbuch der Literatur, Stuttgart ${ }^{61979,} 228$.

ZIMMERMANN, Christiane, Die Namen des Vaters. Studien zu ausgewählten neutestamentlichen Gottesbezeichnungen vor ihrem frühjüdischen und paganen Sprachhorizont (AJEC 69), Leiden 2007. 